\title{
Electromagnetic ELF wave intensification associated with fast earthward flows in mid-tail plasma sheet
}

\author{
J. Liang ${ }^{1}$, B. $\mathrm{Ni}^{2}$, C. M. Cully ${ }^{3}$, E. F. Donovan ${ }^{1}$, R. M. Thorne ${ }^{2}$, and V. Angelopoulos ${ }^{4,5}$ \\ ${ }^{1}$ Dept. of Physics and Astronomy, Univ. of Calgary, Canada \\ ${ }^{2}$ Dept. of Atmospheric and Oceanic Sciences, UCLA, CA, USA \\ ${ }^{3}$ Swedish Institute of Space Physics, Uppsala, Sweden \\ ${ }^{4}$ Institute of Geophysics and Planetary Physics, UCLA, CA, USA \\ ${ }^{5}$ Earth and Space Sciences Department, UCLA, CA, USA
}

Correspondence to: J. Liang (liangj@ucalgary.ca)

Received: 31 October 2011 - Revised: 17 January 2012 - Accepted: 27 February 2012 - Published: 5 March 2012

\begin{abstract}
In this study we perform a statistical survey of the extremely-low-frequency wave activities associated with fast earthward flows in the mid-tail central plasma sheet (CPS) based upon THEMIS measurements. We reveal clear trends of increasing wave intensity with flow enhancement over a broad frequency range, from below $f_{\mathrm{LH}}$ (lower-hybrid resonant frequency) to above $f_{\text {ce }}$ (electron gyrofrequency). We mainly investigate two electromagnetic wave modes, the lower-hybrid waves at frequencies below $f_{\mathrm{LH}}$, and the whistler-mode waves in the frequency range $f_{\mathrm{LH}}<f<f_{\text {ce }}$. The waves at $f<f_{\mathrm{LH}}$ dramatically intensify during fast flow intervals, and tend to contain strong electromagnetic components in the high-plasma-beta CPS region, consistent with the theoretical expectation of the lower-hybrid drift instability in the center region of the tail current sheet. ULF waves with very large perpendicular wavenumber might be Doppler-shifted by the flows and also partly contribute to the observed waves in the lower-hybrid frequency range. The fast flow activity substantially increases the occurrence rate and peak magnitude of the electromagnetic waves in the frequency range $f_{\mathrm{LH}}<f<f_{\mathrm{ce}}$, though they still tend to be short-lived and sporadic in occurrence. We also find that the electron pitch-angle distribution in the mid-tail CPS undergoes a variation from negative anisotropy (perpendicular temperature smaller than parallel temperature) during weak flow intervals, to more or less positive anisotropy (perpendicular temperature larger than parallel temperature) during fast flow intervals. The flow-related electromagnetic whistlermode wave tends to occur in conjunction with positive electron anisotropy.
\end{abstract}

Keywords. Magnetospheric physics (Magnetotail; Plasma sheet; Plasma waves and instabilities)

\section{Introduction}

Fast convective earthward flow, often known as "bursty bulk flow" (BBF) (Baumjohann et al., 1990a; Angelopoulos et al., 1992, 1994; Nagai et al., 1998; Schodel et al., 2001; Runov et al., 2009) is one of the most dynamic features in the magnetotail. The fast flow is known as closely related to various types of waves/turbulences over a broad range of frequency and wavelength. As of this paper's research interest, we shall briefly review a few existing studies that more or less touched the potential relationship between the fast flows and tail plasma waves in the extremely-low-frequency (ELF, $\sim 3 \mathrm{~Hz}-3 \mathrm{kHz}$ ) range. Using IMP8 observations at radial distance $23-46 R_{\mathrm{E}}$ in the tail Gurnett et al. (1976) categorized three main types of plasma waves, broadband electrostatic noises, magnetic noise bursts, and electron cyclotron harmonic $(\mathrm{ECH})$ waves. The authors noticed that many of the broadband electrostatic noise events were accompanied by either earthward- or tailward-streaming ion fluxes, but they did not present detailed statistics in this regard. Also, most of their observations were made in the plasma sheet boundary layer (PSBL) rather than the central plasma sheet. Cattell et al. (1986) presented a few events from ISEE observations in the tail plasma sheet near $\sim 20 R_{\mathrm{E}}$. They found that the intense broadband electrostatic noises and magnetic noise bursts tend to be observed when the DC electric field and the plasma flows were large, while the $\mathrm{ECH}$ waves tend to be observed when the DC electric field and flows were weak. Based upon AMPTE/IRM observations Baumjohann et al. (1989) performed a statistical study on the average electric wave spectra and their dependence on the ion bulk flow, radial distance, and different magnetospheric regions. They 
found that the average electric wave intensities under fast flow condition are considerably higher than those under weak flow condition in all plasma sheet regions, and that the wave intensities during episodes of fast perpendicular (convective) flows are higher than those associated with fast parallel (field-aligned) flows. Using GEOTAIL observations Zhang et al. (1999) confirmed that the "magnetic noise bursts" categorized by Gurnett et al. (1976) are whistler-mode waves, and statistically surveyed those waves over broad tail ranges but mainly within $10-50 R_{\mathrm{E}}$. They found that those whistlermode waves are often narrow-band and short-lived. In two example events shown in their paper the wave bursts were both found as accompanied by fast flows, but the authors did not pursue the statistical relationship between the two phenomena.

The launch of the THEMIS mission (Angelopoulos, 2008) further brought new insights into the conjunction between the fast flows, the plasma sheet dynamics and the ELF wave activities. Le Contel et al. (2009) identified quasi-parallel whistler-mode waves associated with dipolarization fronts and fast flows in an event study. Zhou et al. (2009a, b) identified the ECH and lower-hybrid waves in another event with fast flows and multiple dipolarization fronts. The above two events both occurred in the inner plasma sheet, and were analyzed in the context of substorm dipolarization, though both studies noticed that the occurrence of wave intensification was not exclusively tied to dipolarization fronts only. Deng et al. (2010) presented the observations of whistler-mode wave bursts from two mid-tail THEMIS probes during a BBF event previously reported by Runov et al. (2009), and concluded that the observed whistler-mode waves were associated with the development of electron temperature anisotropy $\left(T_{\mathrm{e} \perp}>\right.$ $\left.T_{\mathrm{e} / /}\right)$ in a flow-driven electron injection process. In an event with a series of fast earthward flows Liang et al. (2011) found one-to-one correspondence between the fast flows and broadband ELF wave intensifications, and proposed that those ELF waves were operational in pitch-angle scattering the mid-tail plasma sheet electrons, which in turn led to the flow-related precipitations detected from the optical and radar observations in the auroral ionosphere.

Despite the above studies, there is still a lack of dedicated and extensive survey on the statistical conjunction between the fast earthward flow (FEF) and the ELF wave activity of both electric and magnetic waves. Such task will be taken in this paper, based upon THEMIS measurements in the midtail central plasma sheet (CPS). Due to our specific research purpose, our data collection and selection differ from previous studies on a number of aspects. (1) In this study we focus our interest in the mid-tail: GSM-X $=-12$ to $-20 R_{\mathrm{E}}$, and $Y=-6$ to $6 R_{\mathrm{E}}$. This delimited region is distinctly separated from the near-geosynchronous plasma sheet where the instability-driven current disruption may also be the cause of fast flows and a variety of wave activities that might complicate the scenario (e.g. see Sigsbee et al., 2002). (2) In this study we limit our research interest to the CPS region (see criterion I in the following section), which is relatively close to the neutral sheet by definition. The plasma waves in the tail are known to have a very complex dependence of different wave modes on the neutral sheet distance and the ambient magnetospheric parameters (Cattell et al., 1986; Baumjohann et al., 1990b). However, we emphasize that our study is not oriented for an investigation on the magnetospheric region dependence of the wave activities, but is exclusively focused on the relationship between the plasma waves and convective FEFs in the CPS region. (3) The dependence of average electric wave spectra on the ion bulk speed was statistically investigated by Baumjohann et al. (1989). However, in their study they used only the electric wave measurement, such that most of their observations and analyses were pertinent to the electrostatic wave modes, e.g. the broadband electrostatic noise and ECH wave. In this study we shall include both the electric and magnetic wave measurements, which allow us to better distinguish and explore the electromagnetic components of the flow-related wave intensification.

The paper is organized as follows. Section 2 introduces the observational instruments and data selection/collection criteria applied in this study. The ELF wave observations in a fast flow event are exemplified in Sect. 3. Section 4 presents the statistical survey of the wave characteristics over $\sim 100$ fast flow events, through which we not only reveal an unambiguous correlation between the FEF and a broad frequency range of ELF waves, but also explore the spectral properties of the flow-related waves, with more focus on the electromagnetic wave modes. In Sect. 5, we discuss some possible underlying mechanisms and the potential roles of the flow-related lower hybrid waves and whistler-mode waves. Section 6 concludes the paper.

\section{Instrumentation and event selection criteria}

Two key THEMIS instruments used in this study are the Electric Field Instrument (EFI, see Bonnell et al., 2008), and the Search Coil Magnetometer (SCM, see Roux et al., 2008). The EFI provides differential electric field measurements, which can be selected as either DC-coupled (EDC) or AC-coupled (EAC), in three directions. The sample rate is 8192/16 384 per second for EDC/EAC channel, corresponding to a Nyquist frequency of $4 \mathrm{kHz} / 8 \mathrm{kHz}$, respectively. The SCM measures the magnetic component of waves in three directions with sample rate up to 8192 per second, covering the frequency band from 0.1 to $4 \mathrm{kHz}$. Based upon the EFI\&SCM measurements two spectral datasets are produced: the FilterBank (FBK) and Fast Fourier Transform (FFT). The FBK data, which is mainly used in this study, represent the mean of the absolute value of the bandpassfiltered signals from the spin-plane EFI\&SCM sensors, in 6 spectra bands. The time resolution of FBK dataset is $4 \mathrm{~s}$. For a complete list of the frequency bins and band widths of the FBK datasets see Cully et al. (2008) and/or the website 
http://themis.ssl.berkeley.edu/beta/dfb_faqs.shtml. Though the FBK data are not ideal in spectral resolution, they provide continuous time coverage and are suitable for an initial survey-type study as in this paper. The FFT dataset contains the FFT power spectrum with up to 64 frequency bins, but is only available during the particle-burst mode intervals. We shall also use the FFT wave data in an example flow event to better demonstrate the representative wave spectra during the FEF interval.

Other onboard instruments applied in this study, mainly for the purpose of event selection, include the Fluxgate Magnetometer (FGM, see Auster et al., 2008), the Electrostatic Analyzer (ESA, see McFadden et al., 2008), the Solid State Telescope (SST). The FGM instrument measures the in-situ magnetic field within the accuracy of $0.01 \mathrm{nT}$. The data used in this study are in spin-resolution $(\sim 3 \mathrm{~s})$. The ESA instrument measures the flux of thermal particles over the energy range from $5 \mathrm{eV}$ to $25 \mathrm{keV}$ for ions, and $6 \mathrm{eV}$ to $28 \mathrm{keV}$ for electrons. The ESA dataset gives full 3-D distributions, as well as the moments such as density, bulk velocity, pressure and temperature, of the ambient electrons and ions. The SST measures the energy flux of superthermal $(\geq 30 \mathrm{keV})$ particles from specific directions. To infer the plasma density we also use the satellite potential obtained from the EFI measurement for reference (data in courtesy of Wen Li, see Li et al., 2010, for technical descriptions).

We survey the THEMIS B/C data over two past tail seasons (2007/12-2008/04 and 2008/12-2009/04) for FEF occurrences in the mid-tail CPS ( $X=-12$ to $-20 R_{\mathrm{E}}, Y=-6$ to $6 R_{\mathrm{E}}$ ). Our event selection criteria are as follows:

(I) Based upon FGM, ESA and SST measurements we select the CPS passage according to plasma- $\beta>1$ which is standard in defining the CPS. We however, impose another requirement, $B_{\mathrm{z}}>\sqrt{B_{\mathrm{x}}^{2}+B_{\mathrm{y}}^{2}}$ (GSM coordinate is used throughout this paper), in our data-collecting procedure. Similar requirement on the magnetic elevation angle is often used to define a so-called "inner CPS", namely a CPS portion in the neighbourhood of the neutral sheet (Angelopoulos et al., 1992; Baumjohann et al., 1989). Certainly, the requirement $B_{\mathrm{z}}>\sqrt{B_{\mathrm{x}}^{2}+B_{\mathrm{y}}^{2}}$ appears be too rigid for a general CPS delimitation, but such requirement is nevertheless imposed in order to relieve two major uncertainties of the FBK data used in this study. Firstly, the FBK data only contain the mean wave field on the spin-plane. Our criterion guarantees a relatively small angle between the ambient magnetic field and the spin-axis. As a matter of fact, we have calculated such angles for all FEF intervals we collected, and found it statistically within $22.6 \pm 12.2^{\circ}$. For many of the wave modes of interest in this study, such as the lower-hybrid drift waves and the whistler-mode waves, the geometry ensures that a substantial component of the electric/magnetic wave field can be recorded by the spin-plane sensor and thus registered in the FBK data. Secondly, our criterion also helps to partly reduce the ambiguity in estimating the B-field strength of the wave source region, which is key to the reorganization of the actual wave mode. Since we have enforced $B_{\mathrm{Z}}$ to be the dominant magnetic field component, under a normal mid-tail configuration and the assumption that the waves owe their sources in the CPS region, the difference between the local magnetic field and the source magnetic field would not be much too significant, particular taking into account the relatively broad bandwidth of the FBK measurements. We admit that the requirement $B_{\mathrm{z}}>\sqrt{B_{\mathrm{x}}^{2}+B_{\mathrm{y}}^{2}}$ certainly implies that our sampled region is somehow smaller than the nominal CPS by more general definitions. This limitation might cause two potential biases of our wave analyses: (a) our data samples are statistically close to the neutral sheet, such that the waves from the neutral sheet sources (e.g. due to temperature anisotropy, Le Contel et al., 2009) likely outweigh the waves from the current sheet boundary layer or PSBL sources (e.g. due to electron beam, Zhang et al., 1993). (b) Our data samples and in turn the wave statistics may have a moderate bias toward dipolarization-front-related FEF intervals (e.g. Runov et al., 2011) against those FEF intervals without significant dipolarization effects.

(II) Our criteria for FEF interval are $E_{\mathrm{y}}>1 \mathrm{mV} \mathrm{m}^{-1}$ and $v_{\mathrm{x}}>140 \mathrm{~km} \mathrm{~s}^{-1}$, with peak $E_{\mathrm{y}}>2 \mathrm{mV} \mathrm{m}^{-1}$ and peak $v_{\mathrm{x}}>$ $200 \mathrm{~km} \mathrm{~s}^{-1} . v_{\mathrm{X}}$ is the $\mathrm{X}$-component of the ion bulk velocity, and $E_{\mathrm{y}}=-(\boldsymbol{v} \times \boldsymbol{B})_{\mathrm{y}}$ is the dawn-dusk convective electric field. The reason that we choose $E_{\mathrm{y}}$ as the main measure of the FEF activity in our event criterion and later in our superimposed statistics is the same as in Schodel et al. (2001). The ion bulk velocity is known to vary with the radial distance in the CPS; such variation is particularly dramatic in our interested tail range -12 to $-20 R_{\mathrm{E}}$. This trend is also identified in our flow dataset (not shown). A number of researchers have proposed and/or actually chose $E_{\mathrm{y}}$, which is less sensitive to the probe location, as a criterion to characterize fast convective flows (Angelopolous et al., 1999; Schodel et al., 2001). Also note that the field-aligned flows and tailward fast flows are excluded by our criterion. This, together with the requirement on $B_{\mathrm{Z}}$ in above criterion (I) as well as the fact that a majority of our events is confined to $X>-18 R_{\mathrm{E}}$, also hereby delimit that our survey region is predominantly earthward of the reconnection site(s). Note that in a practical calculation of the ion moments, a so-called "remote-sensing effect" would arise in the presence of sharp density gradient, which may lead to non-zero first moments without actual bulk plasma motion (e.g. Wilber et al., 2004). However, it is not unreasonable to conceive that sharp density gradients in azimuthal direction might not be statistically common under typical mid-tail configuration. Since our event criteria postulate $B_{\mathrm{Z}}$ as the main magnetic field component, the deviation between the first moments and the bulk plasma velocity in X-direction would not be significant enough to affect our discerning of FEF intervals for statistical purpose.

(III) To evaluate the wave intensification within the FEF interval we need to compare with the waves during the weak 
flow intervals surrounding the FEF. Therefore, for each flow event we count in the data from $15 \mathrm{~min}$ before an individual flow burst or the first of a continuous series of flow bursts (successive flow bursts with separation $<10$ min are considered as a continuous flow series, and are treated as one flow event in this study), to $15 \mathrm{~min}$ after the flow burst or the last of the flow burst series. Of course, any selected data point in the above-delimited interval is still required to be in the CPS (see criterion I).

(IV) FBK data from both EFI and SCM must be available during the flow event. We multiply the raw FBK value by a factor $\pi / 2$ to convert it into the nominal wave amplitude. We resample all the data into 8-s bins, each containing the average of two original FBK data samples and typically three spin-resolution FGM, ESA, and SST data samples. Hereafter, when we refer to a "data point" of our collected wave dataset, we actually mean the rebinned data in an 8-s bin. In the following presentation we abbreviate the 6 FBK bands as FBK0 to FBK5, from high to low frequency ranges, and term "E-wave" and "B-wave" for the EFI and SCM measurements, respectively. The noise detected by SCM is generated by the spacecraft (power circuit and electronics boards) as described by Ludlam et al. (2008) and can be reduced from the SCM waveform as described by Le Contel et al. (2008). The FBK data in each band have certain noise floor. Such noise floor is usually more pronounced in the SCM data (e.g. see the highly clustered baselines of FBK1-FBK4 data in Fig. 4). The EFI noise floor is defined by the minimum quantization level. Such equipment noise floor level certainly has non-negligible influence on our data analyses. For example, the noise floor of the FBK0 band is unfortunately too high, preventing its use in this study. Therefore, we limit our interest to FBK1-FBK5 bands in this paper. The FBK noise floor though, would not seriously interfere with our core research object, namely the flow-related wave intensifications, since such intensifications in general rise well above the noise floor levels. We however have two caveats: (a) the noise floors, particularly in the higher-frequency bands of both EFI and SCM, might mask the low-intensity waves during weak flow intervals; (b) the presence of SCM noise floors may hinder a precise discrimination of the electrostatic and electromagnetic waves in our study.

\section{February 2009 event - an example}

Figure 1 gives a flow event example from $\mathrm{TH}-\mathrm{C}$ observation at $X \sim-18 R_{\mathrm{E}}$. Our main purpose in presenting this event is to demonstrate our data collection/preparation procedure as well as to illustrate a few key observations and calculations that will be used as annotating examples for subsequent statistical investigation. From the top to bottom panels of Fig. 1 are the magnetic fields, ion flow velocities, dawn-dusk convective electric field, plasma- $\beta$, and FBK wave data of EFI and SCM in FBK3 band (20.1-57 Hz centered at $36.2 \mathrm{~Hz}$ ), which is in the whistler-mode frequency range. In collecting the data we first exclude the intervals when the probe was outside the CPS (see criterion I in Sect. 2) or without FBK data, therefore our data series are usually not continuous in time. Then we classify the CPS data points into the three categories: (a) The FEF data points are chosen according to criterion (II); (b) the weak flow background (WFB) data points are selected according to $v<100 \mathrm{~km} \mathrm{~s}^{-1}$ in which $v$ is the flow magnitude, in a time range from up to $15 \mathrm{~min}$ before an individual flow burst or the first of a continuous series of flow bursts, to $15 \mathrm{~min}$ after the flow burst or the last of the flow burst series (see criterion III); (c) in the above-delimited time range, any data point amid the FEF and WFB intervals but not belonging to either FEF or WFB by above definitions, is considered as of "intermediate interval". Such intermediate interval is introduced to buffer the potential arbitrariness in our threshold to separate the FEF and WFB data. The identified FEF, WFB, and intermediate data intervals are marked with different colors in panels 5 and 6 of Fig. 1. Note that the same color code will also be used throughout this paper in presenting our statistical results.

We outline a few key features of our interest as shown in Fig. 1. (1) Correspondence between the E- and B-wave intensifications and the FEFs is straightforward to see, though we do notice that moderate intensification occasionally exists at weak-flow times, e.g. 08:15 UT. (2) Corresponding to individual flow burst the E-wave intensification tends to have a longer duration than the B-wave intensification; the latter is notably more "spiky". (3) The flow burst at 08:08 UT is not accompanied by a dipolarization front, but still corresponds to the strongest among all wave intensifications. This observation hints that the wave intensification may have more general association with FEF than with the dipolarization front only, though there is little doubt that the dipolarization front and the fast flow are often closely related phenomena (e.g. Runov et al., 2009, 2010).

Fortuitously, TH-C was run in particle-burst mode during 07:56-08:06 UT, enabling us to investigate the wave intensifications associated with fast flows in high spectral- and temporal-resolution. In Fig. 2 we present the FFT wave data during this interval from the sensors EDC34 and SCM2 (both on spin-plane). We also present in the top panel the convective electric field $E_{\mathrm{y}}$ to mark the flow burst episodes. One may see from Fig. 2 that, there is a series of E-wave and B-wave intensifications which are in one-to-one correspondence with the series of flow bursts. Those B-wave intensifications are banded within the frequency range $0.1-$ $0.5 f_{\text {ce }}$ and are highly "spiky", each lasting for only a few seconds. They also revealed themselves in the FBK3 data as shown in Fig. 1. In comparison, corresponding to each flow burst the E-wave intensification is more broadband, extending well above $f_{\text {ce }}$, and has a duration comparable to the entire flow burst interval. However, on a close look into those broadband intensifications one might also identify the existence of some embedded narrow-band E-wave "spikes", e.g. at 08:00:14 UT. 


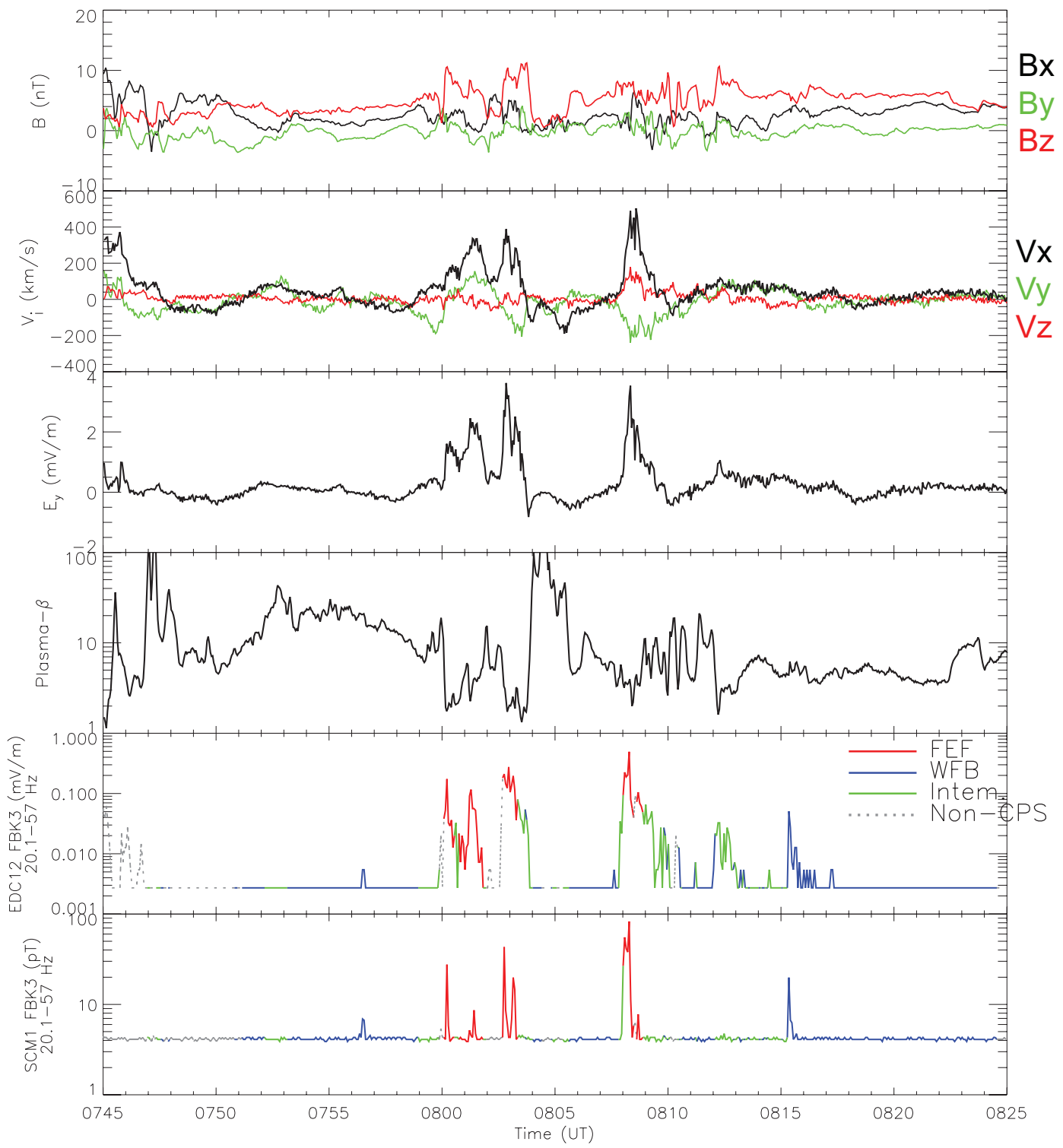

Fig. 1. From top to bottom panels are the magnetic fields in GSM (color code as labelled), ion flow velocities in GSM, dawn-dusk convective electric field, plasma- $\beta$, FBK wave data of EFI and SCM in FBK3 band, from TH-C observations on 5 February 2009. In the bottom two panels the color code marks the FEF (red), WFB (blue), intermediate (green), and non-CPS (gray) intervals which we use to categorize the data.

Panels 4 and 5 in Fig. 2 display the results of wave polarization analysis using the particle-mode SCM (scp) data. Panel 4 shows the wave normal angle spectrogram; a value close to 0 (blue color) corresponding to each wave burst indicates a quasi-parallel propagating wave front. Panel 5 presents the ellipticity in the plane perpendicular to ambient B-field; a value close to 1 (red color) corresponding to each wave burst implies that the wave is right-hand circularly polarized. It thus clear that those wave bursts are quasi-parallel whistler-mode in nature. Such whistler-mode wave bursts were previously reported as existing in the mid-tail (Gurnett et al., 1976; Zhang et al., 1999). It is commonly accepted that the free energy for the growth of whistler-mode waves originates from the pitch-angle anisotropy of the electrons via cyclotron resonance (e.g. Kennel and Petschek, 1966). The minimum resonant energy of such electron cyclotron resonance is given by,

$E_{\text {res }}=\frac{m_{\mathrm{e}} c^{2}}{2} \frac{f_{\mathrm{ce}}^{2}}{f_{\mathrm{pe}}^{2}} \frac{\left(1-f^{\prime}\right)^{2}\left(\cos \theta-f^{\prime}\right)}{f^{\prime} \cos ^{2} \theta}$

in which $f_{\mathrm{pe}}$ is the electron plasma frequency, $\theta$ is the wave normal angle, and $f^{\prime}=f / f_{\text {ce }}$ To make such calculation, we estimate the weighted-average (with the wave power density being the weight factor) frequency of each 


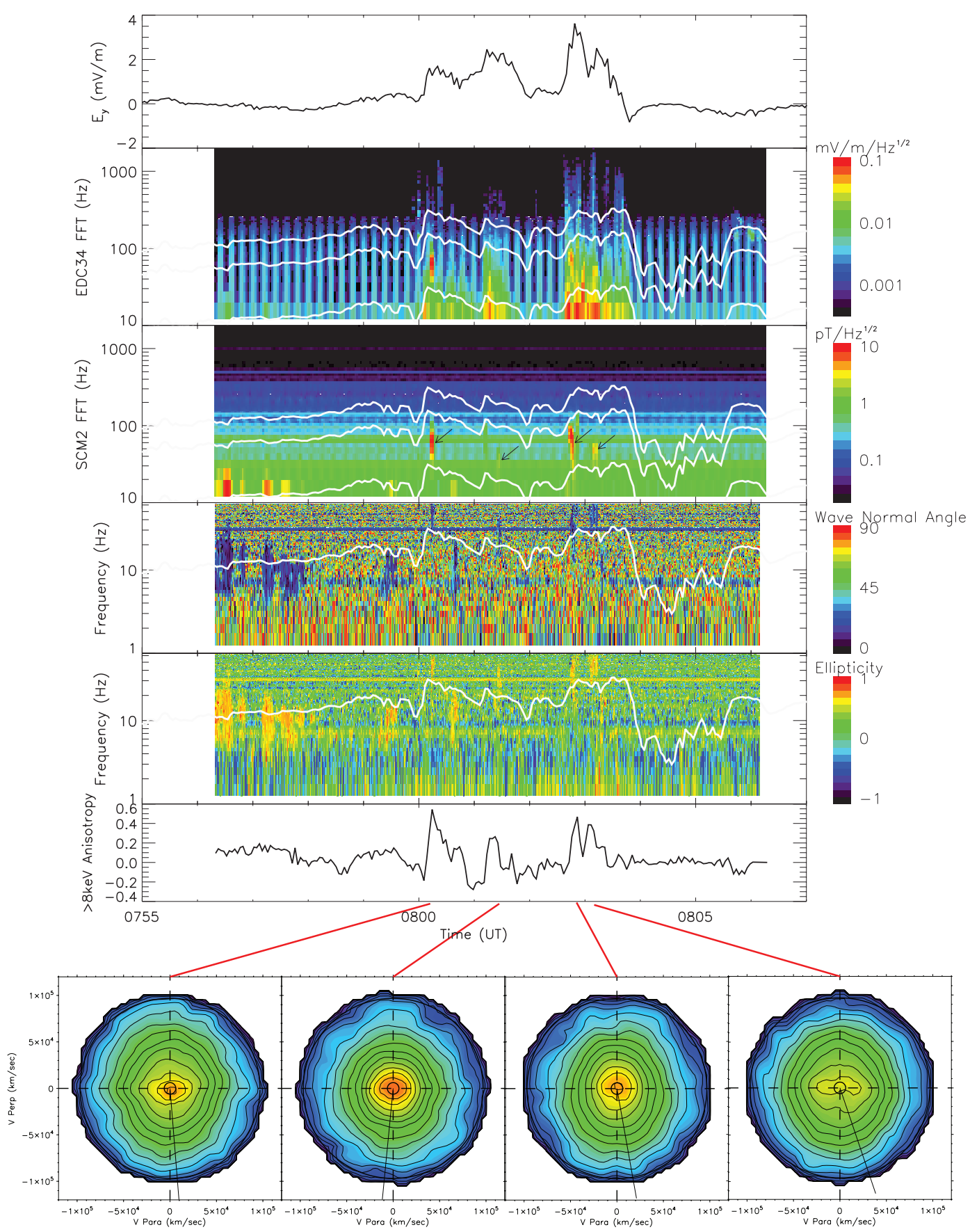

Fig. 2. Top panel shows the dawn-dusk convective electric field. The second and third panels give the FFT spectrograms of E-wave and B-wave form particle-burst mode observations, respectively. We have used four arrows to label our interested B-wave bursts. Three white curves in panels denote $f_{\text {ce }}, 0.5 f_{\text {ce }}$, and $0.1 f_{\text {ce }}$, respectively. The fourth and fifth panels show the wave normal angle spectrogram and wave ellipticity spectrogram from the 3-D particle-burst SCM data, respectively; a white curve in each panel denotes $0.1 f_{\text {ce. }}$ The sixth panel shows the electron anisotropy calculated from Eq. (2). The bottom panel shows four 2-D snapshots of the electron pitch-angle distribution from ESA measurements, corresponding to each peak of the electron anisotropy.

narrow-band whistler-mode wave burst from the FFT data, and the weighted-average wave normal angle from the polarization analysis as shown in panel 4 . The resulting minimum resonant energy corresponding to the four marked wave bursts are 4.6, 2.1, 2.5, and $6.8 \mathrm{keV}$. Without losing the generality, we calculate the anisotropy of $>8 \mathrm{keV}$ electrons according to, 


$$
A=\frac{\int_{0}^{\pi} \tilde{J}(\alpha) \sin ^{3} \alpha d \alpha}{2 \int_{0}^{\pi} \tilde{J}(\alpha) \cos ^{2} \alpha \sin \alpha d \alpha}-1
$$

in which $\tilde{J}(\alpha)$ is the averaged directional energy flux at pitch angle $\alpha$ or $>8 \mathrm{keV}$ electrons, obtained from ESA particleburst mode measurements. See Li et al. (2010) for more details of the definition. $A=0$ corresponds to pitch-angle isotropy. $A>0$ denotes pitch-angle distribution peaking at $90^{\circ}$, while $A<0$ indicates a pitch-angle distribution minimum at $90^{\circ}$. The definition reduces to $A=T_{\mathrm{e} \perp} / T_{\mathrm{e} / /}-1$ for a formal bi-Maxwellian distribution with $T_{\mathrm{e} \perp}$ and $T_{\mathrm{e} / /}$ being the perpendicular and parallel temperature, respectively. The calculated $A$-value is presented in panel 6 of Fig. 2. We see that there is one-to one correspondence among the flow bursts, the electron anisotropy peaks, and the narrow-band whistler-mode wave bursts, though the latter two tend to have timescale much shorter than individual flow bursts. To confirm that the calculated $A$-index indeed reflects the electron anisotropy we also provide the 2-D snapshots of the electron pitch-angle distribution corresponding to each peak epoch of $A$, in the bottom panel of the plot. Pitch-angle anisotropy with stronger energetic electron fluxes in perpendicular direction is evident in every frame. We also note that the intensity of the whistler-mode wave bursts apparently correlates with the anisotropy level. For example, the weakest peak of anisotropy level, namely the one at $\sim 08: 01: 20$ UT, corresponds to the least visible wave burst in this series.

We shall briefly mention another flow event occurring on 27 February $2009 \sim 07: 50$ UT, which was extensively studied from various aspects by Runov et al. $(2009,2010)$, and Deng et al. (2010). Runov et al. (2010) found that on both mid-tail probes (TH-B: $X \sim-20 R_{\mathrm{E}}$, TH-C: $X \sim-17 R_{\mathrm{E}}$ ) the pitch-angle distribution of high-energy electrons became strongly anisotropic with peak flux in the perpendicular direction, in conjunction with the arrival of FEF and dipolarization front. Such strong electron anisotropy was found to be accompanied by intense whistler-wave burst as studied in Deng et al. (2010). Both the strong electron anisotropy and the whistler-mode wave burst were rather short-lived. Their results are highly consistent with our findings in the previous flow event example. As we shall illustrate later, many of the above-revealed features of the flow-related waves in the event study are common in a statistical survey.

\section{Statistical results}

All identified flow events (96 in total) according to our event criteria are listed in Table 1. Figure 3a shows the distribution of the THEMIS probes' positions on GSM-XY plane for all identified flow events. Figure $3 \mathrm{~b}$ gives the scatter plot of the B-field strength versus $E_{\mathrm{y}}$ for all collected data points. As introduced in previous section, we categorize our collected data points into FEF (red), WFB (blue), and intermediate (green). We have in total 1368 FEF data points, 6217 WFB data points, and 1761 data points for intermediate intervals.
For the FEF intervals of our core research interest, we present the statistical histogram of local- $|B|$ in Fig. 3c. The average statistics of $|B|$ is $9.7 \pm 3.8 \mathrm{nT}$. Figure $3 \mathrm{~d}$ illustrates the frequency bands of FBK0 to FBK5, as well as the distribution of two important characteristic frequencies, the lower-hybrid resonant frequency $f_{\mathrm{LH}}$ and the electron gyrofrequency $f_{\mathrm{ce}}$, according to the statistical spread of $|B|$ during FEF intervals as labelled in Fig. 3c. We see that, the lowest band FBK5 is just below $f_{\mathrm{LH}}$ but still much larger than the proton gyrofrequency $f_{\mathrm{ci}}$, such that the data from this band likely represent a lower-hybrid wave mode. FBK4 is on average above, yet may partly cover, $f_{\mathrm{LH}}$. Almost the entire FBK3 and a large portion of the FBK2 are in the nominal whistler-mode frequency range $\left(f_{\mathrm{LH}}<f<f_{\mathrm{ce}}\right)$; FBK1 is essentially above $f_{\text {ce. }}$. The above delimitation can be used as a rough guide in discerning the wave mode of each FBK band in our following presentation.

\subsection{Overall correlation between ELF wave intensifica- tion and flow enhancement}

FBK data from all collected flow events are stacked in Fig. 4. From top to bottom are the data of FBK1 to FBK5 bands, from EFI (left) and SCM (right) measurements. In each subfigure the FBK data of the specific band are superimposed according to the dawn-dusk convective electric field $E_{\mathrm{y}}$. It is straightforward to see that, there are generally unambiguous trends of increasing wave intensity with $E_{\mathrm{y}}$, suggesting a close association between the ELF wave intensification and the fast flow enhancement. The average trends of the wave intensity from FBK1-FBK5 bands as a function of $E_{\mathrm{y}}$ are presented in Fig. 5. Figures 4 and 5 yield key inferences on the ELF wave intensifications for different frequency ranges (and in turn different wave modes). The increase of both $\mathrm{E}$ - and $\mathrm{B}$-wave intensities with $E_{\mathrm{y}}$ is clear-cut in the lowest FBK5 band. In FBK2 and FBK3 bands the increasing trends of the E-waves are also fairly neat, while for the B-waves, though intensifications are also present for some data points, a substantial portion of the data points still concentrate near the SCM noise floors even at strong $E_{\mathrm{y}}$. A reference to the flow event presented in Sect. 3 would help readers to understand the above observation: we showed in Figs. 1 and 2 that $\mathrm{B}$-wave intensifications tend to be bursty, while the E-wave intensifications are more persistent during the FEF intervals. Therefore, when we sample from the FEF intervals and assemble the data into Fig. 4, the occurrence of strong E-wave intensification is understandably much more common than that of the B-wave intensification. For the FBK1 band, an increasing trend of the average wave intensity with $E_{\mathrm{y}}$ is discernible for the E-wave (see Fig. 5) but essentially not for the $\mathrm{B}$-wave. The above-depicted trend in terms of the occurrence rate of visible B-wave intensifications may provide useful inferences in evaluating that the associated wave modes are mainly electromagnetic or electrostatic, as we shall discuss in Sect. 4.2. 
Table 1. Flow event list used in this study.

\begin{tabular}{|c|c|c|c|c|c|c|c|}
\hline Date & Probe & Flow interval (UT) & GSM-X $\left(R_{\mathrm{E}}\right)$ & Date & Probe & Flow interval (UT) & GSM-X $\left(R_{\mathrm{E}}\right)$ \\
\hline 24 Dec 2007 & $\mathrm{C}$ & $04: 45-04: 47$ & -12.6 & 13 Feb 2009 & $\mathrm{C}$ & $08: 40-08: 45$ & -18.0 \\
\hline 28 Dec 2007 & $\mathrm{C}$ & 05:01-05:09 & -13.6 & 13 Feb 2009 & $\mathrm{C}$ & $08: 55-08: 58$ & -18.0 \\
\hline 1 Jan 2008 & $\mathrm{C}$ & $02: 03-02: 07$ & -12.8 & 17 Feb 2009 & $\mathrm{C}$ & $03: 38-03: 48$ & -18.9 \\
\hline 1 Jan 2008 & $\mathrm{C}$ & $04: 46-04: 48$ & -14.2 & 21 Feb 2009 & $\mathrm{C}$ & 05:02-05:09 & -18.5 \\
\hline 1 Jan 2008 & $\mathrm{C}$ & $05: 00-05: 02$ & -14.3 & $21 \mathrm{Feb} 2009$ & $\mathrm{C}$ & $07: 54-07: 59$ & -17.6 \\
\hline 3 Jan 2008 & $\mathrm{C}$ & $03: 19-03: 27$ & -14.0 & 21 Feb 2009 & $\mathrm{C}$ & 10:04-10:27 & -16.4 \\
\hline 5 Jan 2008 & $\mathrm{C}$ & $03: 46-03: 50$ & -14.6 & $21 \mathrm{Feb} 2009$ & $\mathrm{C}$ & $11: 35-11: 37$ & -15.6 \\
\hline 18 Feb 2008 & $\mathrm{C}$ & $07: 32-07: 35$ & -17.5 & 23 Feb 2009 & $\mathrm{C}$ & $09: 12-09: 29$ & -16.6 \\
\hline 20 Feb 2008 & $\mathrm{C}$ & 04:02-04:07 & -18.1 & 23 Feb 2009 & $\mathrm{C}$ & $09: 40-09: 43$ & -16.3 \\
\hline 24 Feb 2008 & $\mathrm{C}$ & $03: 18-03: 27$ & -17.7 & 23 Feb 2009 & $\mathrm{C}$ & $09: 54-09: 55$ & -16.3 \\
\hline 26 Feb 2008 & $\mathrm{~B}$ & $11: 11-11: 37$ & -16.1 & 23 Feb 2009 & $\mathrm{C}$ & $10: 12-10: 13$ & -16.1 \\
\hline 26 Feb 2008 & $\mathrm{C}$ & 05:01-05:06 & -17.1 & 25 Feb 2009 & $\mathrm{C}$ & 00:49-00:50 & -19.0 \\
\hline 26 Feb 2008 & $\mathrm{C}$ & $11: 11-11: 16$ & -14.0 & 25 Feb 2009 & $\mathrm{C}$ & $09: 25-09: 28$ & -16.2 \\
\hline 5 Mar 2008 & $\mathrm{~B}$ & $06: 47-06: 52$ & -18.8 & 27 Feb 2009 & B & 08:07-08:26 & -19.6 \\
\hline 5 Mar 2008 & B & $07: 55-07: 57$ & -17.9 & 27 Feb 2009 & $\mathrm{C}$ & $02: 50-03: 10$ & -18.5 \\
\hline 7 Mar 2008 & $\mathrm{C}$ & $05: 53-06: 03$ & -15.5 & 27 Feb 2009 & $\mathrm{C}$ & 07:01-07:07 & -17.1 \\
\hline 7 Mar 2008 & $\mathrm{C}$ & $06: 29-06: 31$ & -15.3 & 27 Feb 2009 & $\mathrm{C}$ & $07: 52-08: 27$ & -16.4 \\
\hline 9 Mar 2008 & $\mathrm{~B}$ & $05: 57-05: 59$ & -18.6 & 1 Mar 2009 & $\mathrm{C}$ & $01: 43-01: 57$ & -18.6 \\
\hline 11 Mar 2008 & $\mathrm{C}$ & $05: 53-05: 55$ & -14.9 & 3 Mar 2009 & $\mathrm{~B}$ & $06: 36-06: 45$ & -16.5 \\
\hline 11 Mar 2008 & $\mathrm{C}$ & $06: 22-06: 27$ & -14.7 & 3 Mar 2009 & $\mathrm{~B}$ & 06:59-07:13 & -15.9 \\
\hline 13 Mar 2008 & $\mathrm{~B}$ & $07: 27-07: 42$ & -16.2 & 3 Mar 2009 & $\mathrm{C}$ & $03: 31-03: 49$ & -17.8 \\
\hline 13 Mar 2008 & $\mathrm{~B}$ & 08:10-08:12 & -15.8 & 3 Mar 2009 & $\mathrm{C}$ & $06: 17-06: 23$ & -16.6 \\
\hline 13 Mar 2008 & $\mathrm{C}$ & $06: 33-06: 40$ & -14.3 & 3 Mar 2009 & $\mathrm{C}$ & $06: 57-07: 14$ & -16.2 \\
\hline 13 Mar 2008 & $\mathrm{C}$ & $07: 23-07: 42$ & -13.7 & 3 Mar 2009 & $\mathrm{C}$ & $11: 14-11: 18$ & -13.1 \\
\hline 17 Mar 2008 & B & 09:00-09:02 & -14.0 & 5 Mar 2009 & $\mathrm{C}$ & 04:04-04:06 & -17.6 \\
\hline 17 Mar 2008 & B & 09:11-09:26 & -13.7 & 5 Mar 2009 & $\mathrm{C}$ & $04: 22-04: 23$ & -17.5 \\
\hline 17 Mar 2008 & $\mathrm{~B}$ & $10: 23-10: 25$ & -12.7 & 5 Mar 2009 & $\mathrm{C}$ & $08: 13-08: 15$ & -15.4 \\
\hline 21 Mar 2008 & B & $08: 40-08: 42$ & -13.6 & 5 Mar 2009 & $\mathrm{C}$ & 08:51-08:53 & -15.0 \\
\hline 21 Mar 2008 & B & $08: 52-08: 54$ & -13.3 & 7 Mar 2009 & $\mathrm{~B}$ & 03:29-03:31 & -18.8 \\
\hline 21 Mar 2008 & B & 09:11-09:13 & -13.1 & 7 Mar 2009 & $\mathrm{C}$ & $00: 46-00: 56$ & -18.5 \\
\hline 21 Mar 2008 & B & 09:27-09:30 & -12.7 & 7 Mar 2009 & $\mathrm{C}$ & 01:19-01:22 & -18.4 \\
\hline 21 Mar 2008 & $\mathrm{C}$ & $07: 27-07: 32$ & -12.4 & 7 Mar 2009 & $\mathrm{C}$ & 03:03-03:06 & -17.9 \\
\hline 22 Jan 2009 & $\mathrm{C}$ & $03: 30-03: 40$ & -16.2 & 7 Mar 2009 & $\mathrm{C}$ & $03: 31-03: 35$ & -17.7 \\
\hline 26 Jan 2009 & $\mathrm{C}$ & $01: 19-01: 21$ & -16.3 & 9 Mar 2009 & $\mathrm{C}$ & $06: 23-06: 32$ & -16.1 \\
\hline 30 Jan 2009 & $\mathrm{C}$ & $02: 58-03: 15$ & -17.6 & 9 Mar 2009 & $\mathrm{C}$ & $09: 06-09: 23$ & -14.0 \\
\hline 1 Feb 2009 & $\mathrm{C}$ & $03: 56-03: 58$ & -18.0 & 11 Mar 2009 & $\mathrm{C}$ & $02: 18-02: 30$ & -17.7 \\
\hline 3 Feb 2009 & $\mathrm{C}$ & $01: 41-01: 45$ & -17.9 & 11 Mar 2009 & $\mathrm{C}$ & 02:47-03:06 & -17.5 \\
\hline 3 Feb 2009 & $\mathrm{C}$ & $02: 19-02: 25$ & -18.0 & 11 Mar 2009 & $\mathrm{C}$ & $03: 26-03: 35$ & -17.3 \\
\hline 5 Feb 2009 & $\mathrm{C}$ & $05: 28-05: 36$ & -18.5 & 11 Mar 2009 & $\mathrm{C}$ & $09: 13-09: 40$ & -13.5 \\
\hline 5 Feb 2009 & $\mathrm{C}$ & $07: 32-07: 47$ & -18.3 & 13 Mar 2009 & $\mathrm{C}$ & $06: 00-06: 24$ & -15.7 \\
\hline 5 Feb 2009 & $\mathrm{C}$ & 08:00-08:10 & -18.2 & 13 Mar 2009 & $\mathrm{C}$ & $06: 53-07: 26$ & -15.1 \\
\hline 7 Feb 2009 & $\mathrm{C}$ & $04: 05-04: 33$ & -18.6 & 17 Mar 2009 & $\mathrm{C}$ & $05: 45-05: 51$ & -15.5 \\
\hline 7 Feb 2009 & $\mathrm{C}$ & $05: 25-05: 26$ & -18.6 & 17 Mar 2009 & $\mathrm{C}$ & $06: 21-06: 23$ & -15.1 \\
\hline 9 Feb 2009 & $\mathrm{C}$ & $01: 44-01: 48$ & -18.6 & 19 Mar 2009 & $\mathrm{C}$ & $03: 33-03: 35$ & -16.4 \\
\hline 9 Feb 2009 & $\mathrm{C}$ & $01: 58-02: 01$ & -18.7 & 19 Mar 2009 & $\mathrm{C}$ & $07: 50-07: 52$ & -13.8 \\
\hline 11 Feb 2009 & $\mathrm{C}$ & $02: 48-03: 01$ & -18.9 & 19 Mar 2009 & $\mathrm{C}$ & $08: 25-08: 26$ & -13.4 \\
\hline 11 Feb 2009 & $\mathrm{C}$ & 08:43-08:49 & -18.1 & 29 Mar 2009 & $\mathrm{C}$ & $06: 38-06: 40$ & -13.0 \\
\hline 11 Feb 2009 & $\mathrm{C}$ & $10: 43-10: 46$ & -17.5 & 31 Mar 2009 & $\mathrm{C}$ & $05: 22-05: 36$ & -13.4 \\
\hline
\end{tabular}

The start (end) time of the flow interval given in the table denotes the start (end) of an individual flow burst, or that of a continuous series of flow bursts.

Since our data are collected from a relatively broad radial coverage $\left(X=-12\right.$ to $\left.-20 R_{\mathrm{E}}\right)$, it is also interesting to check the radial dependence of the wave intensity and its flow-related intensification. For example, based upon AMPTE/IRM observations Baumjohann et al. (1989) divided the data into two X-distance ranges: $-14 R_{\mathrm{E}}<X<-9 R_{\mathrm{E}}$, 

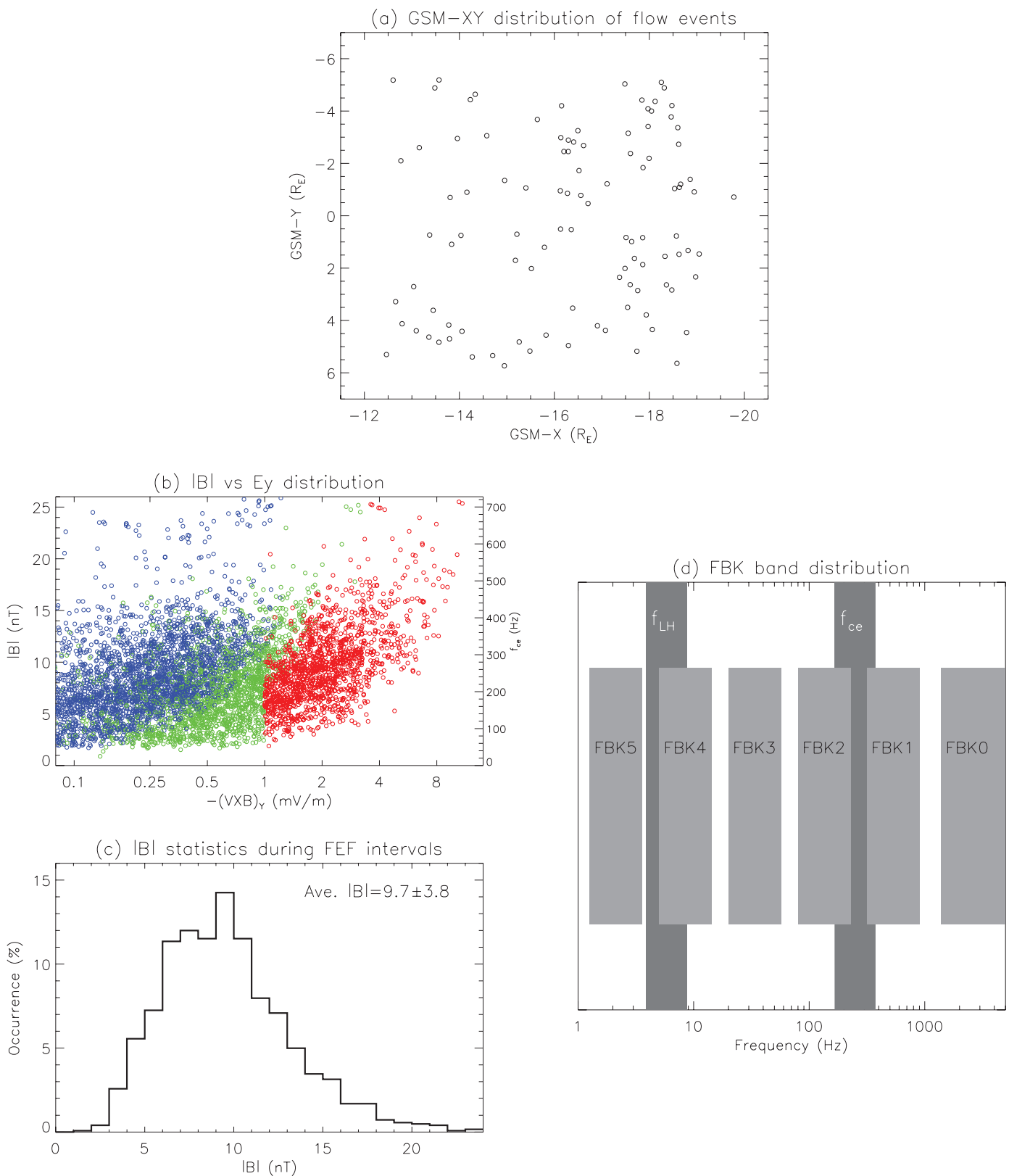

Fig. 3. (a) GSM X-Y distribution of the satellite positions of our identified flow event. (b) Superimposed plot of the magnetic field strength versus the dawn-dusk convective electric field for all flow events. The color code is the same as depicted in Fig. 1. (c) Statistical histogram of B-field magnitude for data points during FEF intervals. (d) The frequency distribution of all FBK bands and two characteristic frequencies, $f_{\mathrm{LH}}$ and $f_{\text {ce. }}$.

and $X<-14 R_{\mathrm{E}}$, and examined the average E-wave spectra in these two tail ranges. However, on a careful reading of their Fig. 3 one may see that the difference of wave intensities at $f<1 \mathrm{kHz}$ between the two tail ranges of the CPS seems not appreciable. To further clarify this issue we also present in Fig. 5 the average trend of E-wave and B-wave intensities in two radial ranges: $-15 R_{\mathrm{E}}<X<-12 R_{\mathrm{E}}$ (dotted lines) and $-20 R_{\mathrm{E}}<X<-15 R_{\mathrm{E}}$ (dashed line). There are more data in the latter range due to the satellite orbit de- sign. We see that the average E-wave intensity tends to be slightly higher in the nearer-Earth radial range; this trend is more discernible when $E_{\mathrm{y}}$ is small, e.g. during weak flow intervals (such trend is not identifiable for B-waves since their intensities are confined to the SCM noise floors at small $E_{\mathrm{y}}$ ). However, the overall differences of the average E-wave and $\mathrm{B}$-wave intensities in two tail ranges seem not significant in terms of their increasing trend with $E_{\mathrm{y}}$. As a matter of fact, in some panels their differences are barely viewable. In all of 


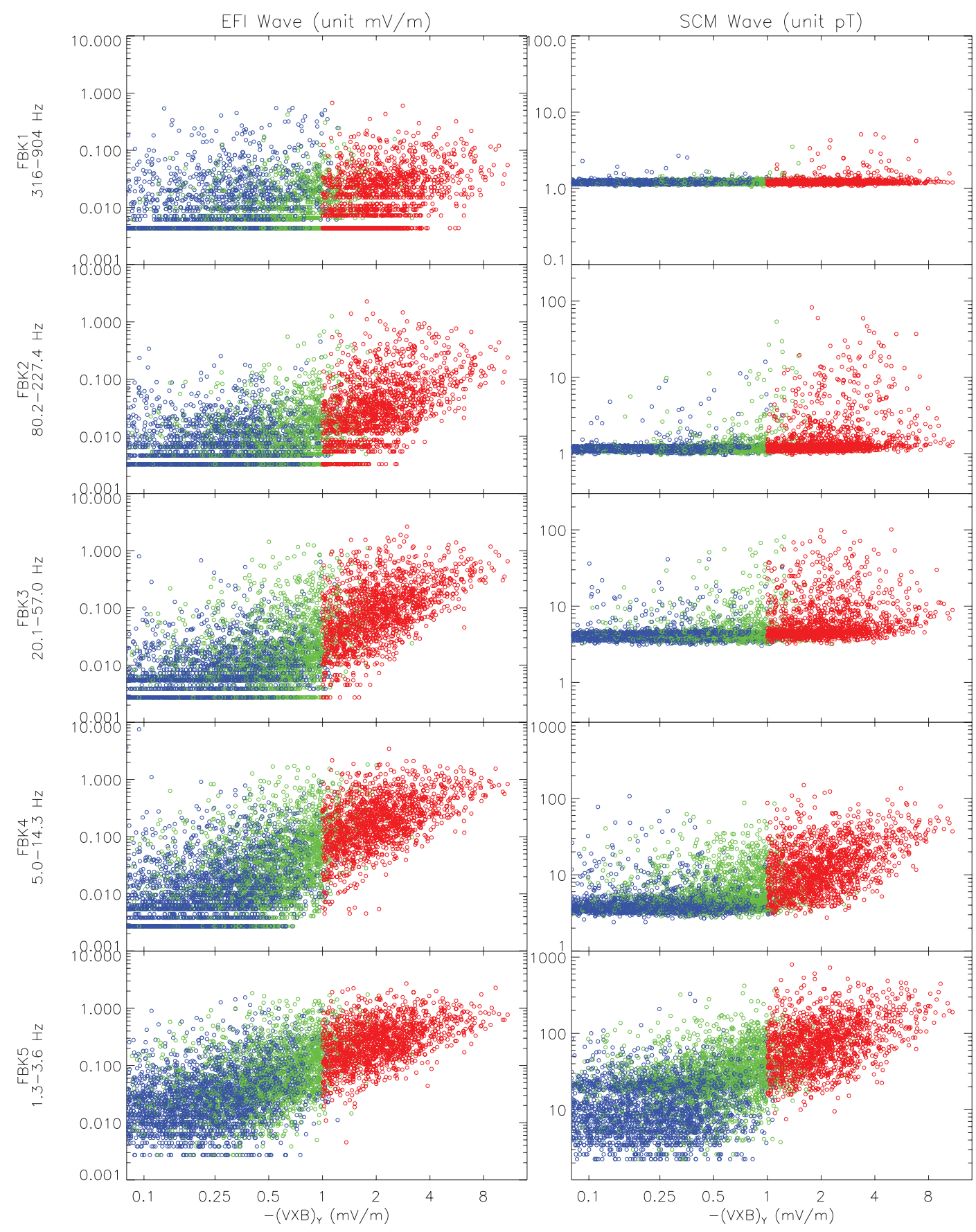

Fig. 4. The scatter plots of FBK wave data of EFI (left) and SCM (right) from 6 FBK bands, versus the dawn-dusk convective electric field. The color code is the same as depicted in Fig. 1.

our following data analyses, we actually make three sets of plots according to the radial distance of measurements: the nearer-tail one $\left(-15 R_{\mathrm{E}}<X<-12 R_{\mathrm{E}}\right)$, the farther-tail one $\left(-20 R_{\mathrm{E}}<X<-15 R_{\mathrm{E}}\right)$, and the combined one. On a careful check of those plots we find that there is no statistically significant and systematic difference in two tail ranges for the wave features of our main interest in this paper, such as the electromagnetic/electrostatic nature, the overall spectral pattern, the whistler-mode wave bursts, etc. Therefore, we will not explicitly differentiate the radial dependence in our following presentation. 


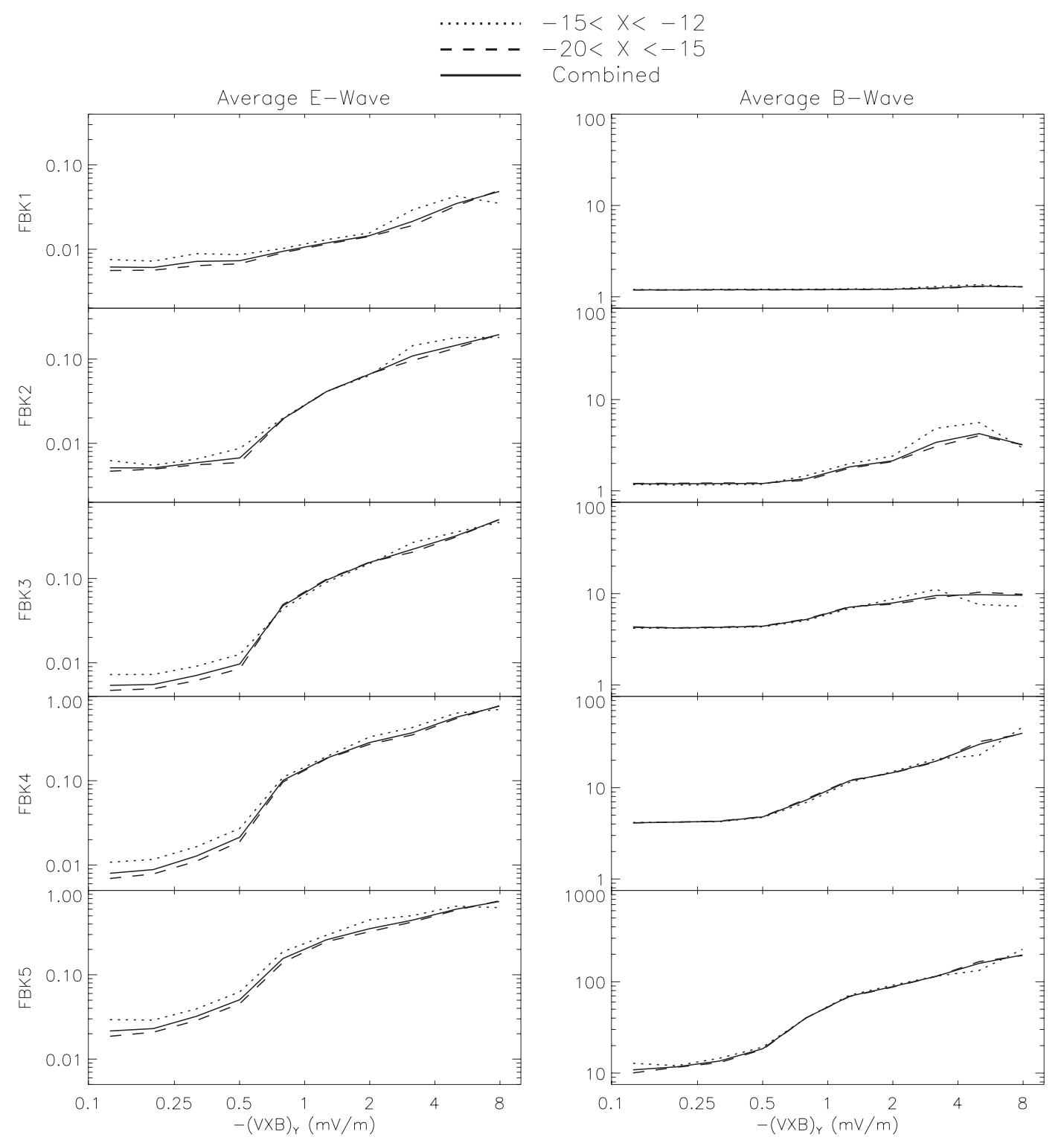

Fig. 5. Variation of average E-wave (left) and B-wave (right) intensity in FBK1 to FBK5 bands, as a function of the dawn-dusk convective electric field. The dotted line, dashed line, and the solid line denote the averages in three different tail ranges, $-15 R_{\mathrm{E}}<X<-12 R_{\mathrm{E}}$, $-20 R_{\mathrm{E}}<X<-15 R_{\mathrm{E}}$, and the combined one $-20 R_{\mathrm{E}}<X<-12 R_{\mathrm{E}}$.

\subsection{Conceptual evaluation of electrostatic and electro- magnetic waves}

The inclusion of both EFI and SCM measurements in this study enables us to evaluate one of the key properties of plasma waves, namely whether the waves are electrostatic or electromagnetic, or a co-existence of the two. For example, strong and neat increases of both E- and B- wave intensities with flow enhancement are clearly shown in FBK5 band, which is just below $f_{\mathrm{LH}}$. The observation indicates that the wave intensification in this band contains strong electromagnetic component. On the other hand, there is little B-wave in- tensification seen in FBK1 band, which is expected since the FBK1 band is essentially above $f_{\text {ce }}$ such that the waves are presumably electrostatic in cold plasma wave theory. Some of the strong B-wave intensifications occasional seen in the FBK1 band are possibly related to the electron phase-space holes. The electron holes typically manifest themselves as electric/magnetic pulse train on in-situ measurements. The duration of individual solitary pulse is often found as on order of $\sim 1 \mathrm{~ms}$ in the magnetotail (e.g. Matsumoto et al., 1994; Andersson et al., 2009), and in turn manifests in spectral data as broadband wave spectrum up to $\mathrm{kHz}$ range. However, as can be seen in Fig. 4, strong B-wave intensifications in the 


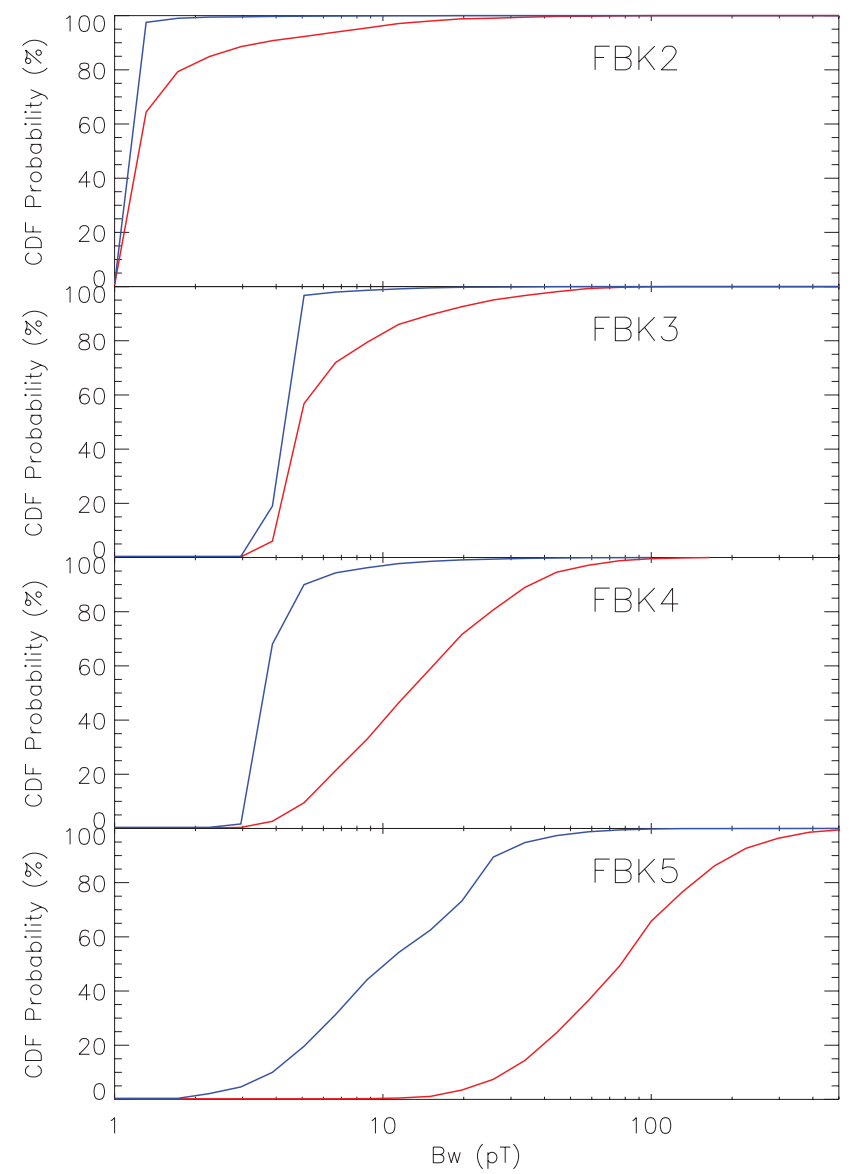

Fig. 6. The cumulative distribution function (CDF) of observed Bwave intensity during WFB (blue) and FEF (red) intervals. From top to bottom panels correspond to FBK2 to FBK5 bands. The CDF corresponding to certain $B_{\mathrm{W}}$ value is calculated as the occurrence percentage of the data points with B-wave intensity smaller than $B_{\mathrm{W}}$. For example, the CDF is $\sim 82 \%$ at $10 \mathrm{pT}$ for FBK3 B-wave during FEF intervals, indicating that $82 \%$ of all FEF data points have B-wave intensity $<10 \mathrm{pT}$ in that band.

FBK1 band are nevertheless rare in occurrence. We conclude that, while there is likelihood that the electron-hole-related wave intensifications indeed exist in occasional cases, their influence on our statistical results may not be appreciable.

The waves in FBK2 to FBK4 bands are more complicated, and likely contain a complex mixture of electrostatic and electromagnetic waves. In this regard, Fig. 6 presents the cumulative distribution function (CDF) of the B-wave measurements from FBK2 to FBK5 bands, for both WFB (blue curves) and FEF (red curves) intervals. The CDF corresponding to certain $B_{\mathrm{w}}$ value is calculated as the occurrence percentage of the data points with B-wave intensity smaller than $B_{\mathrm{W}}$. A steep rise of the $\mathrm{CDF}$, which is the case for the WFB data in FBK2-FBK4 bands, indicates a high concentration of data points at the noise floor level. This trend implies that the actual B-wave intensities during WFB inter-
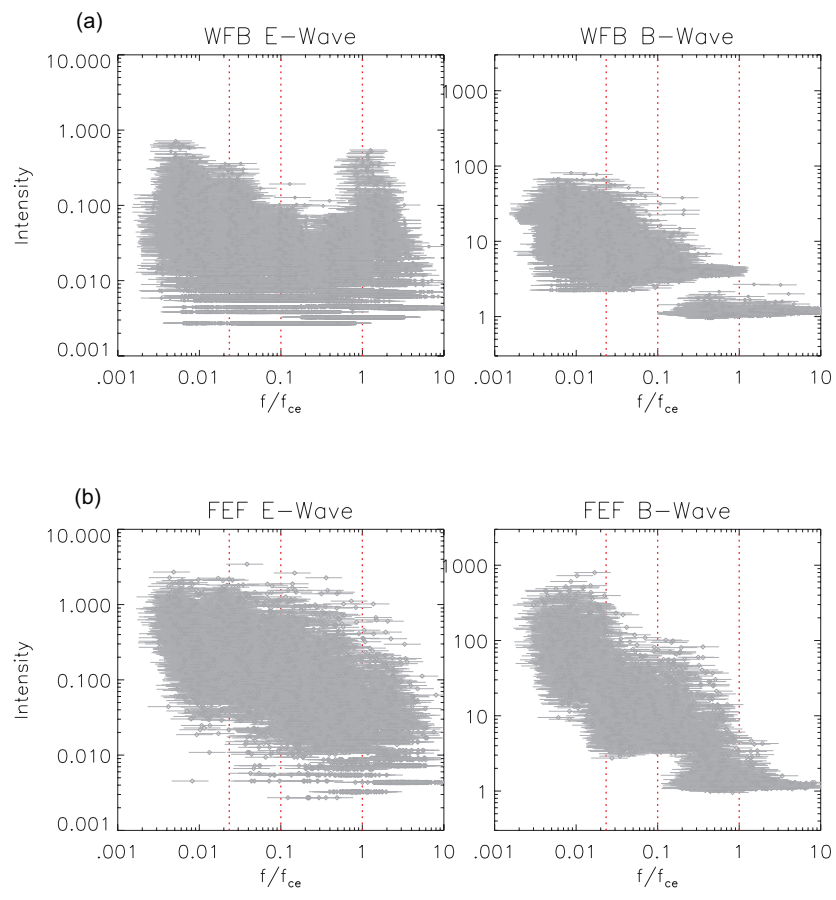

Fig. 7. Panel (a) shows the scatter plot of the FBK wave intensity of E-wave (left) and B-wave (right) versus $f / f_{\text {ce }}$ during WFB intervals. For each data point a diamond (horizontal line) denotes the center frequency (bandwidth) of the FBK measurement. Three vertical red lines denote $f_{\mathrm{LH}}, 0.1 f_{\text {ce }}$, and $f_{\text {ce }}$, respectively. Panel (b) is in the same format as (a) but for FEF intervals.

vals are usually smaller than (or at best equal to) the SCM noise floors. On the other hand, a more gradual rise of the $\mathrm{CDF}$, which is in general the case for FEF data, implies that there are more data points featuring strong B-wave intensifications. Though admittedly a precise discrimination between the electrostatic and electromagnetic waves is unachievable, particularly during WFB intervals, since the SCM noise floor appears to be a little too high in some bands (e.g. FBK3), such ambiguity may not prevent us from making certain qualitative evaluations based upon Figs. 4 and 6: (a) The presence of the FEF substantially increases the occurrence rate and peak magnitude of strong B-wave (electromagnetic) intensifications; (b) The CDF features steeper rise from the noise floor at higher-frequency band. This observation indicates that there is a general trend of decreasing occurrence rate of strong B-wave intensifications, which might imply an increasing prevalence of electrostatic waves, with increasing frequency.

\subsection{Intensity-frequency distribution (spectrum) of flow- related waves}

In this sub-section we shall investigate the spectral properties of the flow-related waves. All WFB and FEF data points are superimposed according to $f / f_{\text {ce }}$ in which $f$ is taken as 

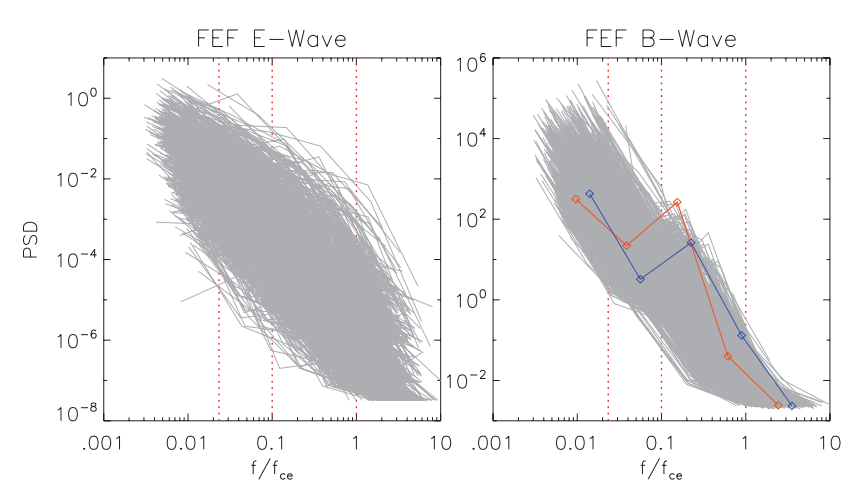

Fig. 8. Superimposed PSD of E-wave (left, in unit of $\mathrm{mV}^{2} \mathrm{~Hz}^{-1}$ ) and $\mathrm{B}$-wave (right, in unit of $\mathrm{pT}^{2} \mathrm{~Hz}^{-1}$ ) during FEF intervals, calculated form the measured FBK wave intensity, the center frequency and spectral width of each FBK band. Coloured curves exemplify the band-limited B-wave spectra of our research interest.

the center frequency of the measuring FBK band. In Fig. 7 we present the distribution of the wave data in the frequencyintensity domain, with information on both center frequencies and bandwidths of the FBK data, during (a) WFB and (b) FEF intervals. Comparing Fig. 7a and b it is straightforward to see that the waves clearly intensify during FEF intervals for almost the entire frequency range, with one exception that during WFB intervals the E-wave distribution shows more "bumps", i.e. local intensity maxima, at frequencies very close to and slightly above $f_{\text {ce }}$. These "bumps" are likely the manifestations of the ECH waves (Cattell et al., 1986; Baumjohann et al., 1989), which fall out of the interest of this paper since we mainly focus on electromagnetic waves at $f<f_{\text {ce }}$. For the FEF wave data of our core research interest, we present in Fig. 8 the superimposed power spectral density (PSD) profiles, calculated from the FBK wave intensity, the center frequency and spectral width of each FBK band. The above plots (Figs. 7 and 8) may appear to be somewhat "messy" due to high volume of data, but a coarse and conceptual view of the general pattern of the E- and B-wave spectra would suffice for our purpose here. The distribution of E-wave intensity is relatively broadband, and generally shows a slow and gradual decrease towards higher frequency. Correspondingly, the overall E-wave PSD appears to be essentially conformal to a power law over a broad frequency range, which is known as the feature of broadband electrostatic noise (e.g. Baumjohann et al., 1989, 1990b). The distribution of the B-wave intensity, on the other hand, is more stepwise-like. Particularly, there is an abrupt bulk drop of B-wave intensity across $f_{\mathrm{LH}}$, which is conformal to the expected decrease of the refractive index in a transition from the lower-hybrid wave to the whistler-mode wave regime.

Comparing Fig. 7a and b one can see that both E- and Bwaves at $f<f_{\mathrm{LH}}$ significantly intensify during FEF intervals, hinting an electromagnetic nature of the lower-hybrid waves. More quantitative analysis of those waves is to be
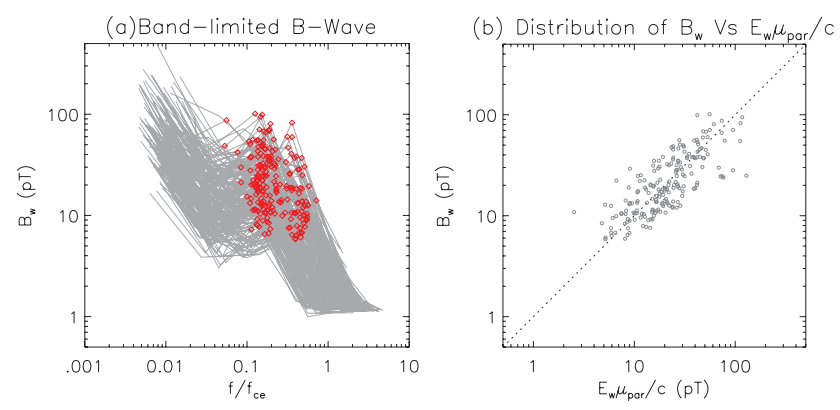

Fig. 9. (a) The superimposed plot of the B-wave intensity versus $f / f_{\text {ce }}$ for the identified band-limited wave structures. Red diamond marks the center frequency of the peak band of each band-limited wave structure. (b) The scatter plot of identified band-limited wave structures, in format of $B_{\mathrm{w}}$ versus $\mu_{\mathrm{par}} E_{\mathrm{w}} / c$ (see text for explanation).

given in next section together with our discussion on existing theories of lower-hybrid drift modes. In this subsection we shall pay more attention to the whistler-mode wave modes in the frequency range $f_{\mathrm{LH}}<f<f_{\text {ce }}$. In particular, we are interested in one special type of wave structures inferred from the FBK spectra. As one can see from Figs. 7b and 8, while the overall trend of B-wave intensity is decreasing toward higher frequency, there are some distinct "bumps", i.e. local intensity maxima, evidently between $\sim 0.1$ to $1 f_{\text {ce }}$ and $\sim 10$ to $100 \mathrm{pT}$ in the distribution. Comparing Fig. $7 \mathrm{a}$ and $\mathrm{b}$ one can see that such "bumps" constitute the most noticeable differences between the WFB waves and FEF waves in the frequency range $f_{\mathrm{LH}}<f<f_{\text {ce }}$. We extract those "bump" structures from the FEF B-wave data according to the criterion that the wave intensity at certain FBK band is $>2.5$ times higher than the geometric average of the wave intensities in two neighbour (above and below) FBK bands. Note that the FBK bands are logarithmically equispaced with scaled bandwidths (i.e. $\Delta f / f \approx$ const.). Our identified structure would point to the existence of a coherent wave structure with limited spectral width, out of a background noise/turbulence. We term such occurrences as "band-limited" structures. Two such example spectra are shown in Fig. 8. Figure 9a shows the superimposition of identified band-limited B-wave structures during the FEF intervals; the center frequency of each identified band-limited structure is highlighted with red diamond. We see that those band-limited structures are mainly distributed in the frequency range $0.1-0.6 f_{\text {ce }}$, and intensity range $10-100 \mathrm{pT}$. To confirm those band-limited B-waves as electromagnetic whistler-mode one needs to compare the observed wave characteristics to the theoretical dispersion relationship. In a cold plasma wave theory, a whistler-mode wave with wavevector parallel to the ambient magnetic field $\left(\boldsymbol{k} \times \boldsymbol{B}_{0}=0\right)$ is purely electromagnetic; its E-wave and Bwave intensities follow the dispersion relationship, 


$$
\frac{E_{\mathrm{w}}}{B_{\mathrm{w}}}=\frac{c}{\mu_{\mathrm{par}}}
$$

where $\mu_{\mathrm{par}}=\sqrt{1+f_{\mathrm{pe}}^{2} / f\left(f_{\mathrm{ce}}-f\right)}$ is the refractive index of parallel-propagating whistler-mode wave. Whistler-mode waves with oblique wave normal angle $\left(\boldsymbol{k} \times \boldsymbol{B}_{0} \neq 0\right)$ contain electrostatic components (i.e. $\boldsymbol{k} \cdot \boldsymbol{E}_{\mathrm{w}} \neq 0$ ), and their $E_{\mathrm{w}} / B_{\mathrm{w}}$ ratio may differ substantially from $c / \mu_{\mathrm{par}}$. We present in Fig. 9b the distribution of $B_{\mathrm{w}}$ versus $\mu_{\mathrm{par}} E_{\mathrm{w}} / c$ for those band-limited wave events. We have used the local density and magnetic measurements, and apply the center frequency of the FBK band in calculating $\mu_{\mathrm{par}}$. We caution that the FBK data only provide the mean wave amplitude on the spin-plane, such that the measured $E_{\mathrm{w}} / B_{\mathrm{w}}$ does not reflect the full polarization, though our data selection criterion (I) (see Sect. 2) ensures that the spin-axis does not significantly deviate from the ambient magnetic field direction and thus helps to reduce the uncertainty to some degree. Also, the finite bandwidth of the FBK measurement introduces further uncertainty to the estimation of $\mu_{\mathrm{par}}$. Despite the above instrumental limitations, one can see that the distribution shown in Fig. $9 \mathrm{~b}$ is fairly close to the condition $E_{\mathrm{w}} / B_{\mathrm{w}} \sim$ $c / \mu_{\mathrm{par}}$. More quantitatively, the ratio $\mu_{\mathrm{par}} E_{\mathrm{w}} / c B_{\mathrm{w}}$ is found be dominantly within $0.5-2$, i.e. a factor of 2 around the theoretical expectation for parallel-propagating whistler-mode wave. We thus propose with certain confidence that the observed band-limited B-wave structures likely represent quasiparallel whistler-mode waves. On a more detailed investigation into those band-limited B-waves, we also find that those band-limited wave events are sporadic and short-lived. They exist in only $\sim 14 \%$ of all FEF data points, each typically surviving only 1 or 2 consecutive data bins. Such statistics are of course somewhat contingent upon our criterion to identify the band-limited structure, but this ambiguity may not seriously affect our statement on the sporadic and bursty nature of those band-limited wave events. In an event study presented in Sect. 3 we have shown, with higher spectral and temporal resolution, clear examples of spiky, narrow-band whistler-mode wave bursts embedded in the FEF interval. Certainly, whistler-mode wave intensification might occur as well during WFB intervals. For example, Fig. 2 shows a few whistler-mode wave bursts at $f \sim 0.1 f_{\text {ce }}$ around $\sim 07: 57 \mathrm{UT}$, without accompanying fast flows. We however point out that, in statistical sense strong whistler-mode wave intensifications during WFB intervals have much lower occurrence rate, and tend to feature smaller peak magnitudes, than those during FEF intervals, which can be inferred from a direct comparison between Fig. 7a and b, or alternatively via the statistical CDFs of B-wave intensifications in FBK2/FBK3 bands as shown in Fig. 6. Though the whistler-mode wave bursts (also known as "magnetic noise bursts") were previously recognized as existing in the tail plasma sheet (e.g. Gurnett et al., 1976; Zhang et al., 1999), in this study we for the first time statistically confirm a close relationship be- tween the FEF activities and the whistler-mode wave bursts, in that the former substantially raise the occurrence rate and the peak magnitude of the latter.

\subsection{Electron anisotropy and its relationship to whistler- mode wave}

Pitch-angle anisotropy of ambient electrons provides the free energy source of many known ELF/VLF wave modes, such as the whistler-mode and ECH waves. In this sub-section we shall investigate the overall temperature anisotropy and the anisotropy of energetic electrons, including their evolution with the flow enhancement and their relationship to the observed wave intensity. For this research purpose, we have carefully made an event-by-event check of the ESA flux data quality in all angular bins to ensure their reliability in calculating the anisotropy. A small number of flow events are excluded from the following statistics in this regard. The top panel of Fig. 10 presents the scatter plot of the overall electron temperature anisotropy, defined as $A=T_{\mathrm{e} \perp} / T_{\mathrm{e} / /}-1$, versus $E_{\mathrm{y}}$. The distribution shows a general bias of $A<0$ during weak flow intervals. However, with the enhancement of $E_{\mathrm{y}}$ the trend is somewhat reversed, i.e. there are more data points at $A>0$. A more accurate approach to analyze the relationship between the whistler-mode waves and the electron anisotropy would be first calculating the minimum resonant energy of the electron cyclotron resonance for specific waves, and then computing the anisotropy of electrons above this minimum resonant energy. Unfortunately, the minimum resonant energy is heavily sensitive to the exact wave frequency and wave normal angle, both of which cannot be precisely determined from the FBK measurement. In an event study with high-spectral resolution particle-burst measurement as presented in Sect. 3, we estimate that the minimum electron resonant energy is typically several for the observed whistlermode wave bursts. In a statistical survey of whistler-mode waves from GEOTAIL measurements Zhang et al. (1999) obtained an average resonant energy of $11 \mathrm{keV}$ but with considerable spread. Without losing the generality, we consider the anisotropy of $>10 \mathrm{keV}$ electrons from ESA measurements for demonstration purpose in this paper. The bottom panel of Fig. 10 shows the electron anisotropy of $10-30 \mathrm{keV}$ electrons as defined by Eq. (2), based upon ESA reduced-mode (peer) measurements. We see that the trend of variation from $A<0$ to $A>0$ with the flow enhancement becomes more evident and better-defined for $>10 \mathrm{keV}$ electrons, though there is always a substantial portion of data points clustering around $A \approx 0$. Similar to the argument applied to the B-wave intensification, we infer that the occurrence of strongly positive $A$ during FEF intervals also tends to be bursty and sporadic, as can be seen from the example event shown in Fig. 2. Considering the uncertainty of the minimum resonant energy we have also checked the anisotropy in a broader energy range 1-30 keV (not shown), and found that the above-revealed trends are essentially unchanged. 


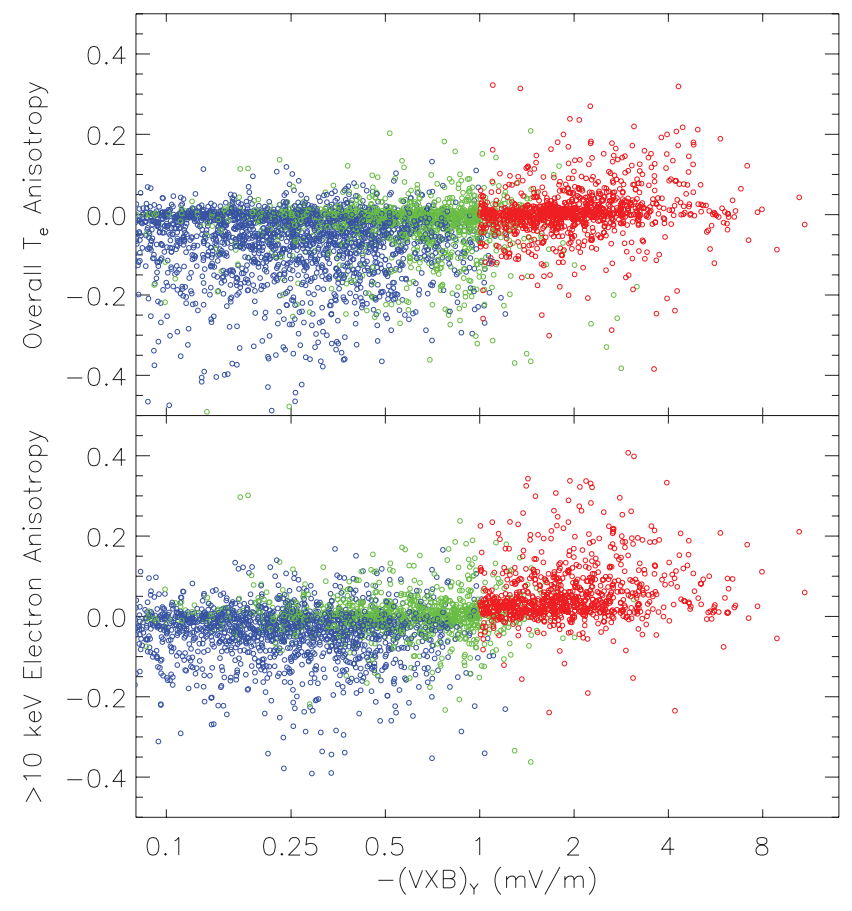

Fig. 10. (a) The scatter plot of the overall temperature anisotropy versus the dawn-dusk convective dawn-dusk electric field. The color code is the same as depicted in Fig. 1. (b) Similar to (a) except that the anisotropy is calculated for the $10-30 \mathrm{keV}$ electrons according to Eq. (2).

To check the dependence of the wave intensity on the anisotropy level, we present in Fig. 11 the scatter plot of Bwave intensities from FBK2 and FBK3 bands, which dominantly cover the whistler-mode frequency range, versus the above-obtained anisotropy of $>10 \mathrm{keV}$ electrons. We see that, the B-wave intensities essentially concentrate at the noise floors for negative anisotropy, implying that the electromagnetic whistler-mode waves are usually weak under such condition. On the other hand, stronger $B_{\mathrm{w}}$ data points during FEF intervals (marked as red color) tend to occur in conjunction with positive anisotropy, though both intense $B_{\mathrm{w}}$ and large anisotropy are notably sporadic in occurrences, consistent with our previous analyses.

The electron anisotropy shown in Figs. 10 and 11 appears to be exclusively smaller than $\sim 0.4$; one may question that whether such anisotropy level be strong enough to account for the observed whistler-mode waves. A much detailed calculation in this regard, such as done in Le Contel et al. (2009), is beyond the scope of the paper. We shall put forth a few qualitative evaluations on the capability of the electron anisotropy in producing the observed whistler-mode waves. (1) The threshold electron anisotropy to excite the whistler anisotropy instability (WAI) varies inversely with the parallel electron- $\beta$ value (Gary and Wang, 1996). Referring to the model and observation in Le Contel et al. (2009),

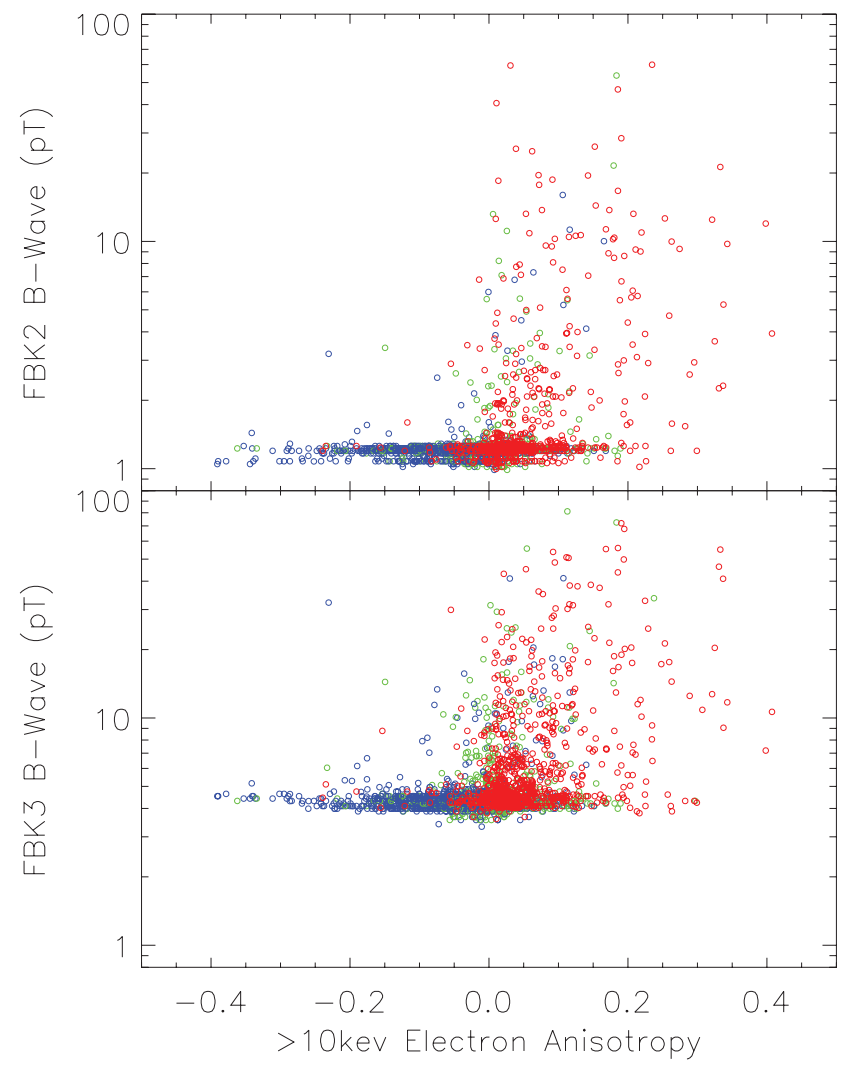

Fig. 11. The scatter plot of the B-wave intensity in FBK2 and FBK3 bands, versus the $>10 \mathrm{keV}$ electron anisotropy. The color code is the same as depicted in Fig. 1.

when the parallel electron- $\beta$ is around $1, A \sim 0.4$ is found as sufficient to excite strong whistler-mode waves. Due to our event criteria our collected CPS data points in general feature relatively large electron- $\beta$ values, mostly ranging between 1-5. Therefore, it is reasonable to speculate that, even with the moderate levels of the observed anisotropy the WAI might actually be excitable. (2) In this statistical study we have used the reduced-angular-resolution observation mode to achieve better time coverage and temporal resolution, but the anisotropy is likely underestimated due to the very coarse angular resolution (only 6 angular bins). As a matter of fact, in a few events with particle-burst mode observations we do achieve larger anisotropy from high-angularresolution measurements, e.g. as exemplified in Fig. 2, and also see Deng et al. (2010). (3) The electron temperature anisotropy tends to decrease with distance to the neutral sheet (Le Contel et al., 2009). Therefore, even though the locallymeasured electron anisotropy might not be particularly high, the anisotropy might be sufficient to excite the whistler instability at the neutral sheet which is deemed as the wave source region in this context. We admit that our obtained values of electron anisotropy may not be subject to a strictly quantitative check on its capability of exciting the observed 
whistler-mode waves. Nevertheless, our revealed trends that the electron anisotropy changes from being dominantly negative during weak flow intervals to more or less positive during FEF intervals, and that the flow-related B-waves tend to occur in conjunction with $A>0$, are well-defined and noncontrovertible.

\section{Discussion}

In above sections we have presented event example as well as statistical survey of ELF wave activities in association with FEFs in the mid-tail CPS. In this section we shall further discuss a few key properties and the underlying mechanisms of the flow-related intensifications of two electromagnetic wave modes, the lower-hybrid waves and whistlermode waves, based upon available observations and theoretical considerations.

\subsection{Lower-hybrid waves}

"Lower-hybrid wave" often refers to the wave regime in the frequency range $f_{\mathrm{ci}} \ll f<f_{\mathrm{LH}}$. For typical mid-tail parameters the lower-hybrid waves are usually at the lowest end of the ELF range, but they may play key intermediate role on the excitation of higher-frequency ELF waves. For example, the lower-hybrid instability mode can modify the velocity distribution of electrons and thus may lead to favorable conditions for a number of electron-kinetic-scale instability modes (Karimabadi et al., 2004). Also, the wave collapse at the lower hybrid resonance involving fast magnetosonic and lower-hybrid waves offers a mechanism for wave pumping to short wavelengths (Sagdeev et al., 1977). The lowest-frequency band FBK5 $(1.3-3.6 \mathrm{~Hz})$ is statistically right below $f_{\mathrm{LH}}$ as shown in Fig. 3. The observations in Figs. 4 and 5 reveal that both the E-wave and B-wave in this band show strong and neat increases with the flow enhancement, implying that the wave intensification contains a strong electromagnetic component. We argue that such wave intensification is likely a consequence of the lower-hybrid drift instability (LHDI) in the tail current sheet. Classical LHDI is often known as purely electrostatic and localized at the edge of the current sheet; it was supposed to be stabilized by either high plasma- $\beta$ (Davidson et al., 1977) or the magnetic normal $\left(B_{\mathrm{Z}}\right)$ component (Pritchett and Coroniti, 2001) in the center region of the current sheet. Such traditional scenario though, is modified by a number of recent developments on the theories and observations of the LHDI. Shinohara et al. (1998) observed the electromagnetic waves in the lower-hybrid frequency range near the neutral sheet, and analyzed the destabilization of the LHDI in the presence of $B_{\mathrm{Z}}$ component taking into account the effect of magnetic field curvature. In a kinetic simulation Daughton (2003) showed that for the fastest-growing LHDI with wavelength on the electron gyroscale, the resulting mode struc-

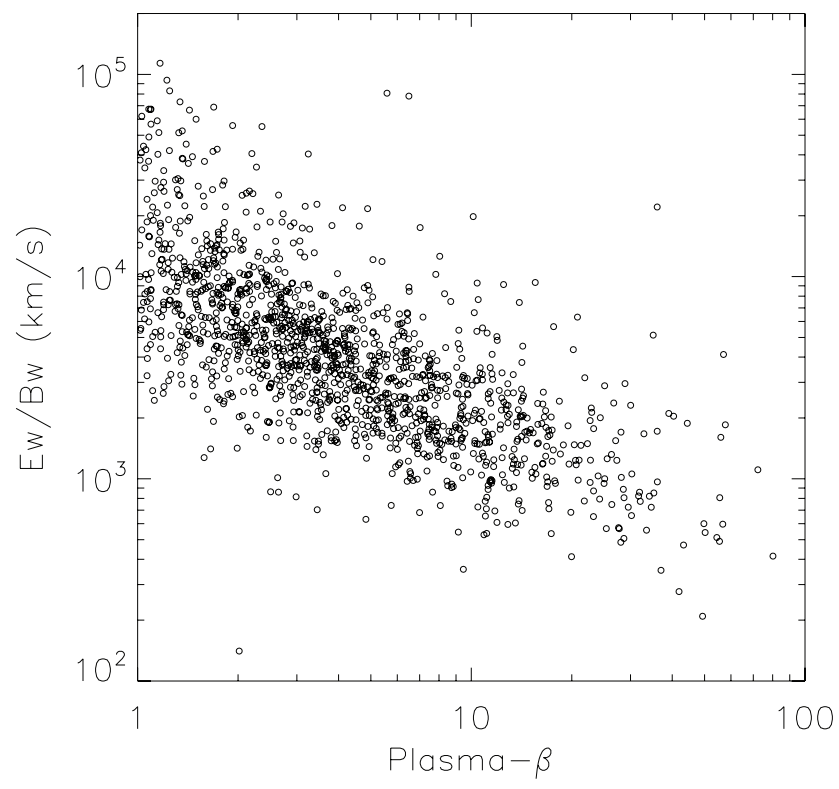

Fig. 12. The scatter plot of $E_{\mathrm{W}} / B_{\mathrm{W}}$ ratio of lower-hybrid waves at $f<f_{\mathrm{LH}}$ during FEF intervals, versus the plasma- $\beta$.

ture is mainly localized on the edge of the current sheet and is quasi-electrostatic. However, for modes with wavelength intermediate between the electron and ion gyroscales, the LHDI has a significant electromagnetic component and is localized in the center region of the current sheet. This scenario is supported by the observations of Zhou et al. (2009a) near a reconnection region with thin current sheet at $X \sim-18 R_{\mathrm{E}}$. Sitnov et al. (2004) studied the current-driven instability in forced current sheets. Their obtained unstable mode resembles the long-wavelength LHDI mode studied by Daughton (2003) in many aspects, except that the new mode is less structured across the sheet as compared to Daughton (2003)'s mode. Though their current sheet model was without $B_{\mathrm{Z}}$, Sitnov et al. (2004) argued that their obtained LHDI-like mode might nevertheless survive in the presence of finite $B_{\mathrm{z}}$. Ji et al. (2005) proposed another generalization of LHDI in the high- $\beta$ region of current sheet, namely the LHDI with wavevector oblique to the ambient B-field, to explain the observations of obliquely-propagating electromagnetic waves in the lower-hybrid frequency range in the center region of a laboratory current sheet from the Magnetic Reconnection Experiment. To summarize, there are growing evidences that certain generalized LHDI modes, which posses strong electromagnetic component, might exist in the center region of the current sheet with high- $\beta$ and/or $B_{\mathrm{Z}}$-component.

The above theories and observations in general lead to the scenario that the LHDI tends to be electrostatic at the boundary of the current sheet, yet electromagnetic in the center of the current sheet. To test such notion, in Fig. 12 we present the scatter plot of $E_{\mathrm{w}} / B_{\mathrm{W}}$ ratio of the FEF waves at $f<f_{\mathrm{LH}}$ ( $f$ is approximated by the center frequency of 
the FBK bands), dominantly from FBK5 measurements, versus the plasma- $\beta$. We caution again that the FBK data contain only the mean electric and magnetic field components on the spin plane. Also, in the present study only data at $\beta>1$ are collected. Nevertheless, Fig. 12 reveals a clear trend that the $E_{\mathrm{w}} / B_{\mathrm{w}}$ ratio decreases with increasing $\beta$, consistent with the expectation that there is a gradual transition from electrostatic waves at the boundary of the current sheet (low $\beta$ ) to electromagnetic waves near the center of the current sheet (high $\beta$ ). More quantitatively, the electromagnetic LHDI mode near the center of the current sheet is expected to feature a wavenumber $k \sim\left(\sqrt{\rho_{\mathrm{i}} \rho_{\mathrm{e}}}\right)^{-1}$ (Daughton, 2003) in which $\rho_{\mathrm{i}}$ and $\rho_{\mathrm{e}}$ denote the gyroradii of the thermal ion and electron, respectively. Assuming typical CPS values $T_{\mathrm{i}} \sim 6 \mathrm{keV} ; T_{\mathrm{e}} \sim 1 \mathrm{keV} ; B \sim 10 \mathrm{nT}$ inferred from our FEF dataset, and applying the center frequency of FBK5 band, the phase velocity of the electromagnetic LHDI wave is estimated as $\sim 1000 \mathrm{~km} \mathrm{~s}^{-1}$, which is compatible with the observed $E_{\mathrm{w}} / B_{\mathrm{w}}$ ratios in the high- $\beta$ region $(\beta>10)$. The above observations and theoretical considerations lead us to propose that the observed waves at $f<f_{\mathrm{LH}}$ likely mainly represent the LHDI modes with certain mixture of electrostatic and electromagnetic components, contingent upon the probe's location with respect to the neutral sheet as expected from LHDI theories.

We acknowledge that a trend of deceasing $E_{\mathrm{w}} / B_{\mathrm{w}}$ ratio with increasing plasma- $\beta$ is not unique to lower-hybrid drift waves. For example, Alfvén waves can also have phase speed and in turn $E_{\mathrm{w}} / B_{\mathrm{w}}$ ratio larger in the PSBL and smaller in the neutral sheet. More detailed analysis and justification of the LHDI mode will be implemented in a separate study (J. Liang, manuscript in preparation) exclusively focusing on the lower-hybrid waves, in which we allow for broader plasma- $\beta$ range and investigate the full 3-D wave field data in fast-survey mode using the flow event list used in this paper.

Lower-hybrid wave intensifications associated with fast flows are also identified in a number of previous studies. Cattell and Mozer (1986) reported intense lower-hybrid wave intensifications associated with tailward fast flows when a near-Earth neutral line was believed to form earthward of the satellite $\left(X \sim-20 R_{\mathrm{E}}\right)$. Sigsbee et al. (2002), and Zhou et al. (2009b) all identified strong lower-hybrid wave intensifications in association with fast flows and dipolarization fronts in the near-Earth CPS $(L \sim 10-13)$. We thus suggest that the conjunction between the fast flow and the LHDI is common over a broad range of the tail plasma sheet, though the detailed underlying linkage between them remains to be answered in future. The growth of LHDI in the tail current sheet during FEF intervals may have an important consequence of the LHDI lies in that its non-linear evolution may lead to a quick perpendicular heating of the electrons and in turn the development of electron anisotropy (Daughton et al., 2004), a condition conducive to the excitation of WAI, which we shall discuss later in this section.
There is however, one other potential source of the observed electromagnetic waves in the FBK5 band. Since such band $(1.3-3.6 \mathrm{~Hz})$ is in a transition from nominal ULF to ELF regime, it is conceivable that some of the observed waves are contributed by lower-frequency ULF waves/turbulences, particularly when they are Doppler-shifted by fast flows. Two kinetic effects may arise in a current sheet with finite $B_{\mathrm{z}}$. The first one is associated with the bounce motion of particles under mirror point reflection (e.g. Liu and Liang, 2009; Le Contel et al., 2000), which mainly affects the waves with frequencies smaller than the particle bounce frequency. The particle bounce frequency for a magnetic bottle geometry is approximately $B_{\mathrm{Z}} V_{\text {th }} / 2 B_{0} L$, in which $V_{\text {th }}$ is the particle's thermal speed, $B_{0}$ is the lobe B-field strength which can be estimated from local measurements as $B_{0} \approx \sqrt{2 \mu_{0} P_{\mathrm{th}}+B_{\mathrm{x}}^{2}+B_{\mathrm{y}}^{2}}$ under the assumption of pressure balance, and $L$ is the half-thickness of the current sheet. Using the realistic observed plasma parameters in our FEF dataset, and assuming $L \sim 1 R_{\mathrm{E}}$, we estimate that the average bounce frequency is about $\sim 0.6 \pm 0.2 \mathrm{~Hz}$ for the mid-tail CPS electrons (the ion bounce frequency is even much smaller), which is below the FBK5 band. We therefore infer that the kinetic effect associated with the particle's bounce motion may not heavily affect the wave regime of our interest. The second kinetic effect is often known as "finite ion-Larmor-radius effect" whose characteristic frequency is typically on order of the gyrofrequency $f_{\mathrm{ci}}$. ULF wave perturbations at frequencies near and/or above $f_{\mathrm{ci}}$ are indeed often observed in conjunction with fast flows and magnetic dipolarizations in the tail CPS. Using Cluster observations Volwerk et al. (2003) investigated the statistical PSD properties of ULF compressional waves associated with fast flows in the mid-tail. They obtained an average PSD of $\sim 1 \mathrm{nT}^{2} \mathrm{~Hz}^{-1}$ at $f=0.1 \mathrm{~Hz}$, and an average spectral index of $\sim 2.9$ in the frequency range $0.08-1 \mathrm{~Hz}$ ( $1 \mathrm{~Hz}$ is their instrumental Nyquist frequency) which was interpreted as a quasi-2-D turbulence. If such turbulence spectrum extended into higher frequency range, it would predict a PSD of the order of $\sim 100 \mathrm{pT}^{2} \mathrm{~Hz}^{-1}$ at the center frequency of FBK5 band $(\sim 2.3 \mathrm{~Hz})$. Comparing to the observed PSDs in the FBK5 band (see Fig. 8), we conclude that our observed waves are unlikely contributed by a higher-frequency extension of the flow-driven ULF turbulence studied in Volwerk et al. (2003). One other type of ULF wave activity extensively studied in conjunction with fast flows and substorm intensifications is the current-driven Alfvén wave with frequencies of the order of $f_{\text {ci }}$ (Perraut et al., 2000, 2003; Le Contel et al., 2001, 2002). Using GEOTAIL observations in the mid-tail Perraut et al. (2003) found that such current-driven Alfvén waves notably intensified with fast flows. The current-driven Alfvén waves typically have most of their energy at $f<f_{\mathrm{ci}}$, but their spectrum may extend well above $f_{\text {ci }}$ (Perraut et al., 2000). Such spreading of the spectrum is likely caused by a well-known Doppler-shift effect: the lower-frequency waves convecting with fast flows may be Doppler-shifted to higher 


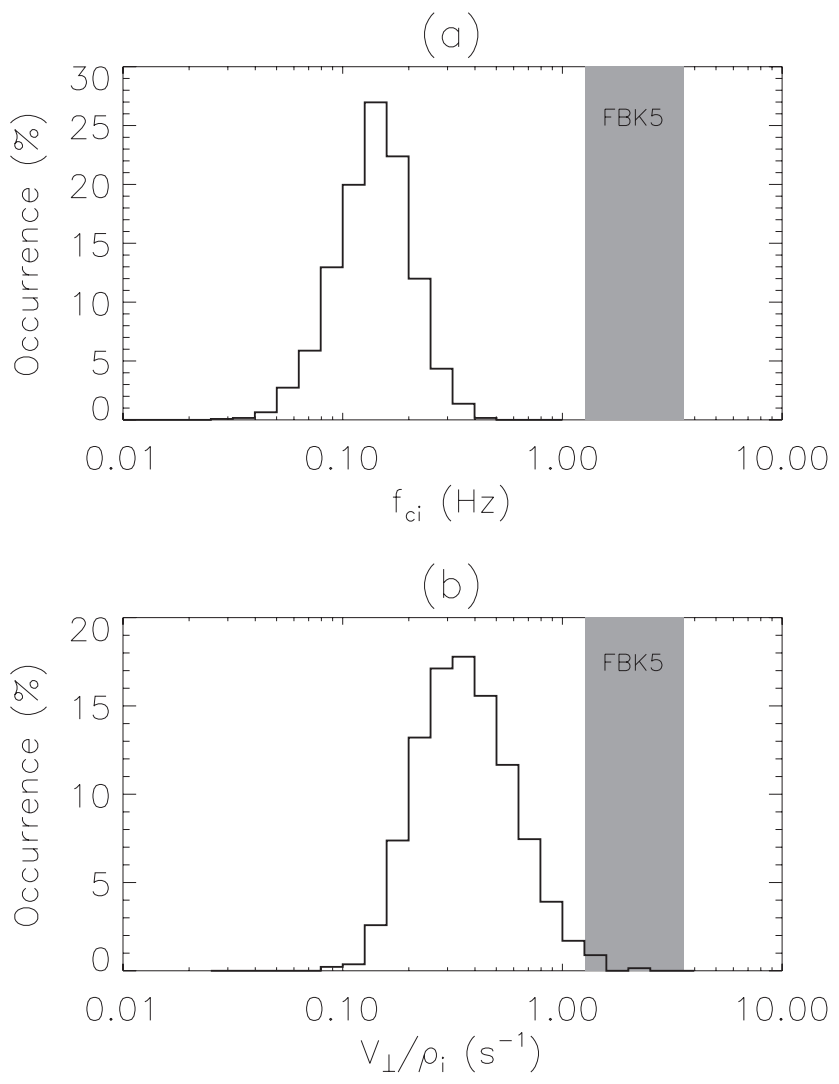

Fig. 13. (a) Statistical histogram of $f_{\text {ci }}$ for data points during FEF intervals. (b) Statistical histogram of $v_{\perp} / \rho_{\mathrm{i}}$ for data points during FEF intervals. In both (a) and (b) the FBK5 band is shown as gray bar.

frequencies and appear as extending spectrum in the satellite frame.

To check whether the above-mentioned characteristic wave modes at $f \sim f_{\text {ci }}$ might considerably contribute to the waves measured in FBK5 band, we present in Fig. 13a the statistical histogram distribution of the local $f_{\text {ci }}$ from our FEF dataset. We see that the entire distribution of $f_{\text {ci }}$ is about an order smaller than the FBK5 band. Therefore, without substantial Doppler-shift the ULF waves whose main spectral energy lies at frequencies around and/or below $f_{\text {ci }}$ might not heavily contribute to the FBK5 measurements. The Doppler-shift effect imposed by the convective flows is difficult to accurately evaluate, since such effect depends on the perpendicular wavenumber $k_{\perp}$ of the wave along the flow direction, which cannot be properly estimated from our data. In many theoretical treatments of the waves involving ion gyro-kinetic effect, $k_{\perp}$ is often normalized with the thermal ion gyro-radii $\rho_{\mathrm{i}}$. It is thus instructive to check the statistical distribution of $v_{\perp} / \rho_{\mathrm{i}}$ from the FEF dataset, in which $v_{\perp}$ denotes the perpendicular flow component (close to $v_{\mathrm{x}}$ due to our FEF criteria), and $\rho_{\mathrm{i}}$ is calculated according to the observed ion perpendicular temperature and B-field. As shown in Fig. 13b, the average statistics of $v_{\perp} / \rho_{\mathrm{i}}$ is $0.4 \pm 0.2 \mathrm{~s}^{-1}$, which is again much smaller than the center frequency of the FBK5 band $(\sim 2.3 \mathrm{~Hz})$. Therefore, in statistical sense, for lower-frequency waves to be effectively Doppler-shifted by fast flows into the FBK5 band, large $k_{\perp} \rho_{\mathrm{i}}(>\sim 30)$ is required. Such small-perpendicular-scale wave certainly exists in both theories and observations. In particular, the shortscale kinetic Alfvén wave is well-known as capable of featuring very large $k_{\perp} \rho_{\mathrm{i}}$ (Howes et al., 2008; Roux et al., 2011). Sahraoui et al. (2009) analyzed the B-wave spectra measured in the solar wind and interpreted the observed fluctuations as small-scale kinetic Alfvén waves. They suggested that the kinetic Alfvén wave can propagate over a wide range of scales before being damped at the electron gyroscale. We admit that such small-scale kinetic Alfvén waves Dopplershifted by fast flows certainly exist in our measurements, and partly contribute to the observed waves in the lower-hybrid frequency range.

\subsection{Whistler-mode waves}

The flow-related electromagnetic waves in the whistler-mode frequency range $\left(f_{\mathrm{LH}}<f<f_{\mathrm{ce}}\right)$ and their relation to the evolution of electron anisotropy constitute the other key new results in this study. We first argue that a majority of our observed whistler-mode waves are not heavily susceptible to a Doppler-shift alias from lower-frequency wave/turbulence, according to the following observations and considerations. (1) As seen in Fig. 7b there is a sharp bulk-drop of the Bwave intensities across $f_{\mathrm{LH}}$; the associated PSD features a spectral slope of about -4.2 across $f_{\mathrm{LH}}$ as estimated from Fig. 8. Such slope is much steeper than that of almost all recognized and theoretically investigated lower-frequency electromagnetic turbulences Doppler-shifted by the flows (see, e.g. the introduction in Sahraoui et al., 2009, and references therein). We suggest that such drop of B-wave intensity across $f_{\mathrm{LH}}$ is more liable to be interpreted as a mode transition from a lower-hybrid to a whistler-mode wave regime when an abrupt decrease of the refractive index is expected. (2) We have shown evidences in Fig. 9b that, the most pronounced manifestations of the B-wave intensification in the frequency range $f_{\mathrm{LH}}<f<f_{\text {ce }}$, namely the band-limited wave structures, roughly comply with the expected dispersion relationship of quasi-parallel whistler-mode waves.

We have illustrated in Fig. 10 that the electron distribution in the mid-tail CPS undergoes a transition from negative anisotropy $\left(T_{\mathrm{e} \perp}<T_{\mathrm{e} / /}\right)$ during weak flow intervals to more or less positive anisotropy $\left(T_{\mathrm{e} \perp}>T_{\mathrm{e} / /}\right)$ during fast flow intervals. The flow event example shown in Sect. 3, as well as that in Runov et al. (2010), clearly demonstrated the emergence of strong anisotropy of energetic electrons accompanying the FEFs. The flow-related whistler-mode wave intensification is very likely to be associated with such developing anisotropy of energetic electrons in the mid-tail CPS. The negative anisotropy during weak flow intervals can be 
understood as follows. In a typical outer magnetosphere configuration, when an electron convects earthward and traverses the mid-tail CPS, the shortening of field line length is more dramatic than the increase of the equatorial magnetic field. Therefore, the electron would undergo more Fermi acceleration than betatron acceleration. Such $T_{\mathrm{e} \perp}<T_{\mathrm{e} / /}$ condition is known as unfavorable to excite the WAI, and thus may explain the minimal whistler-mode wave activities during weak flow intervals. The positive $A$ during FEF intervals may owe its generation to a few possible mechanisms. (1) The rapidly-increasing magnetic fields within the dipolarization front associated with the FEF naturally lead to a betatron acceleration that enhances the perpendicular flux of the electrons (Asano et al., 2010; Ashour-Abdalla et al., 2011). Asano et al. (2010) suggested that the energetic electrons typically undergo more betatron acceleration than Fermi acceleration during a dipolarization front passage, hereby producing the positive anisotropy. (2) The nonlinear evolution of an LHDI, whose development with fast flows can be inferred from the FBK5 band measurements, can cause a perpendicular heating of the electrons and in turn lead to $T_{\mathrm{e} \perp}>T_{\mathrm{e} / /}$ within the current sheet (Daughton et al., 2004; Karimabadi et al., 2004; Le Contel et al., 2009). (3) High-energy electron beam with very strong pitch-angle anisotropy may also contribute to the overall anisotropy as calculated from Eq. (2), even though the electron beam itself is typically only a small portion of the ambient plasma. Such electron beamplasma system is known as capable of exciting the electrostatic waves and electromagnetic whistler-mode waves over a broad range of frequency and wave normal angle, and was theoretically investigated by many researchers (e.g. Omura and Matsumoto, 1987; Zhang et al., 1993). There is so far a lack of confirmative observations on the exact origin of the high-energy electron beam in the magnetotail, but it is often speculated as related to a reconnection activation region (e.g. Zhang et al., 1999), which is also commonly deemed as the origin of the FEF. Under the above notion the electron beam might not have a direct relationship to the FEF itself, but they are likely co-existing. To summarize, the development of electron anisotropic in the CPS can be originate from both local and non-local sources, and involve both adiabatic and non-adiabatic processes. Those processes and sources are potentially closely related to the fast flows and/or the reconnection activities (e.g. Ashour-Abdalla et al., 2011).

We have noticed that strong whistler-mode waves are usually bursty and sporadic in occurrence during FEF intervals. One plausible interpretation to this observation lies in that, while the flow enhancement gives rise to the development of the electron anisotropy as discussed above, the concurrently intensified electrostatic waves may, on the other hand, quickly diffuse the electrons and reduce their pitch-angle anisotropy, and in turn suppress the growth of the whistlermode waves. As a consequence of such suppression process by the electrostatic wave, both the electron anisotropy and the whistler-mode wave intensification tend to be short-lived as compared to the electrostatic wave. This scenario was proposed to explain the bursty nature of whistler-mode waves observed by GEOTAIL in Zhang et al. (1999) and is also applicable to our observations.

We shall end our discussion with a comment on the potential role of the observed flow-related ELF waves in pitchangle scattering the mid-tail CPS electrons, which in turn may result in the auroral precipitations accompanying the fast flows. The role of ELF/VLF waves in producing diffuse auroras in the inner magnetosphere is well known and extensively studied. Our observed wave intensities in the midtail are in general smaller than those reported in the inner magnetosphere (e.g. Li et al., 2009; Meredith et al., 2009). However, the efficiency of the pitch-angle diffusion process is determined by the parameter $Z=D_{\alpha} T_{\mathrm{b}} / \alpha_{\mathrm{c}}^{2}$, in which $D_{\alpha}$ is the diffusion coefficient, $T_{\mathrm{b}}$ is the escaping time for a particle in the equatorial loss-cone to precipitate into the Earth's atmosphere, and $\alpha_{\mathrm{c}}$ is the equatorial loss-cone angle. In a typical mid-tail configuration the field line is often stretched with smaller $B_{\text {eq }}$, such that the diffusion coefficient required to reach a strong diffusion limit is significantly reduced as compared to that in the inner magnetosphere. The notable effect of stretched field-line configuration on the pitch-angle diffusion of CPS electrons by whistler-mode chorus has been recently investigated by $\mathrm{Ni}$ et al. (2011). Based upon the T89 magnetic field model with $\mathrm{Kp}=2$, and the common assumption that the diffusion coefficient is proportional to the wave power, we infer that the wave amplitude required for a strong diffusion limit decreases by about an order of magnitude from the inner magnetosphere $(L \sim 6)$ to the mid-tail $(L \sim 15)$ for the same electron energy range. Therefore, it is likely that the observed flow-related wave intensification is strong enough to effectively pitch-angle scatter the mid-tail CPS electrons and lead to their precipitations into the Earth's ionosphere. Both electrostatic waves and electromagnetic waves may contribute to this scattering process. Though the observed whistler-mode waves appear to be sporadic from single probe measurements, it is not unreasonable to speculate that those waves might be extensively distributed over the entire fast flow region, and stochastically contribute to the pitch-angle scattering of CPS electrons, which in turn produce large-scale auroral features accompanying the fast flows (Kepko et al., 2009; Liang et al., 2011).

\section{Summary and conclusion}

In this paper, we present a THEMIS survey on the ELF wave activities in the mid-tail CPS during FEF intervals. By using the FBK datasets of both EFI and SCM measurements over a large number of flow events, we confirm an intimate correlation between the FEF and the ELF wave intensification in the mid-tail CPS. We discuss the possible underlying mechanisms and the potential roles of those flow-related ELF 
waves, with more focus on the electromagnetic wave modes. Main results in this study include:

1. The statistical results reveal clear trends of increasing wave intensity with flow enhancement over a broad frequency range, from below $f_{\mathrm{LH}}$ to above $f_{\text {ce }}$.

2. Both E-wave and B-wave in the frequency range $f_{\mathrm{ci}} \ll$ $f<f_{\mathrm{LH}}$ show neat and strong intensifications with flow enhancement. We mainly attribute such wave intensification to the growth of the LHDI in the mid-tail current sheet. We find that, for those waves their $E_{\mathrm{w}} / B_{\mathrm{w}}$ ratios decrease with increasing plasma- $\beta$, consistent with the theoretical expectation of the LHDI in different regions of the tail current sheet. We also acknowledge that ULF waves with very small perpendicular scale (large $k_{\perp} \rho_{\mathrm{i}}$ ), such as the kinetic Alfvén waves, might be Dopplershifted by the fast flows and partly contribute to the observed waves in the lower-hybrid frequency range.

3. The FEF activity substantially increases the occurrence rate and the peak magnitude of the electromagnetic waves in the frequency range $f_{\mathrm{LH}}<f<f_{\mathrm{ce}}$, though they still tend to be short-lived and sporadic in occurrence. We particular investigate the band-limited electromagnetic wave structures during FEF intervals, and verify that they are composed of quasi-parallelpropagating whistler-mode waves.

4. We illustrate that the electron anisotropy in the midtail CPS undergoes a variation from dominantly negative $\left(T_{\mathrm{e} \perp}<T_{\mathrm{e} / /}\right)$ during weak flow intervals to more or less positive during FEF intervals. The flow-related electromagnetic waves in the whistler-mode frequency range tend to occur in conjunction with the positive anisotropy. In an event study, we reveal one-toone correspondence among the fast flows, the electron anisotropy, and the whistler-mode wave bursts. The observations are consistent with the common notion that the electron pitch-angle anisotropy provides the free energy source for the growth of whistler-mode waves. Such electron anisotropy may owe its development to both adiabatic and non-adiabatic processes, which may all be related to the fast flow activities.

Acknowledgements. We acknowledge NASA contract NAS502099 for the THEMIS Mission. Specifically: J. W. Bonnell and F. S. Mozer for use of EFI data; A. Roux and O. Le Contel for the SCM data; C. W. Carlson and J. P. McFadden for use of ESA data; K. H. Glassmeier, U. Auster and W. Baumjohann for the use of FGM data provided under the lead of the Technical University of Braunschweig and with financial support through the German Ministry for Economy and Technology and the German Center for Aviation and Space under contract 50 OC 0302; We are grateful to W. Li, V. Uritsky, O. Santolik, and A. Runov for helpful discussions.

Topical Editor R. Nakamura thanks K. Snekvik and another anonymous referee for their help in evaluating this paper.

\section{References}

Andersson, L., Ergun, R. E., Tao, J., Roux, A., Le Contel O., Angelopoulos, Bonnell, J., McFadden, J. P., Larson, D. E., Eriksson, S., Johansson, T., Cully, C. M., Newman, D. L., Goldman, M. V., Glassmeier, K.-H. , and Baumjohann, W.: New features of electron phase space holes observed by the THEMIS mission, Phys. Rev. Lett., 102, 225004, doi:10.1103/PhysRevLett.102.225004, 2009.

Angelopoulos, V.: The THEMIS Mission, Space Sci. Rev., 141, 534, doi:10.1007/s11214-008-9336-1, 2008.

Angelopoulos, V., Baumjohann, W., Kennel, C. F., Coroniti, F. V., Kivelson, M. G., Pellat, R., Walker, R. J., Lühr, H., and Paschmann, G.: Bursty bulk flows in the inner central plasma sheet, J. Geophys. Res., 97, 4027-4039, 1992.

Angelopoulos, V., Kennel, C. F., Coroniti, F. V., Pellat, R., Kivelson, M. G., Walker, R. J., Russell, C. T., Baumjohann, W., Feldman, W. C., and Gosling, J. T.: Statistical characteristics of bursty bulk flow events, J. Geophys. Res., 99, 21257-21280, 1994.

Angelopoulos, V., Mozer, F. S., Lin, R. P., Mukai, T., Tsuruda, K., Lepping, R., and Baumjohann, W.: Comment on "Geotail survey of ion flow in the plasma sheet: Observations between 10 and 50 RE” by W. R. Paterson et al., J. Geophys. Res., 104, 17521, 1999.

Asano, Y., Shinohara, I., Retinò, A., Daly, P. W., Kronberg, E. A., Takada, T., Nakamura, R., Khotyaintsev, Y. V., Vaivads, A., Nagai, T., Baumjohann, W., Fazakerley, A. N., Owen, C. J., Miyashita, Y., Lucek, E. A., and Rème, H.: Electron acceleration signatures in the magnetotail associated with substorms, J. Geophys. Res., 115, A05215, doi:10.1029/2009JA014587, 2010.

Ashour-Abdalla, M., El-Alaoui, M., Goldstein, M. L., Zhou, M., Schriver, D., Richard, R., Walker, R., Kivelson, M. G., and Hwang, K.-J.: Observations and simulations of non-local acceleration of electrons in magnetotail magnetic reconnection events, Nat. Phys., 7, 360-365, doi:10.1038/nphys1903, 2011.

Auster, H. U., Glassmeier, K. H., Magnes, W., Aydogar, O., Baumjohann, W., Constantinescu, D., Fischer, D., Fornacon, K. H., Georgescu, E., Harvey, P., Hillenmaier, O., Kroth, R., Ludlam, M., Narita, Y., Nakamura, R., Okrafka, K., Plaschke, F., Richter, I., Schwarzl, H., Stoll, B., Valavanoglou, A., and Wiedemann, M.: The THEMIS fluxgate magnetometer, Space Sci. Rev., 141, 235-264, doi:10.1007/s11214-008-9365-9, 2008.

Baumjohann, W., Treumann, R. A., LaBelle, J., and Anderson, R. R.: Average Electric Wave Spectra Across the Plasma Sheet and Their Relation to Ion Bulk Speed, J. Geophys. Res., 94, 1522115230, doi:10.1029/JA094iA11p15221, 1989.

Baumjohann, W., Paschmann, G., and Lühr, H.: Characteristics of the high-speed flows in the plasma sheet, J. Geophys.Res., 95, 3801-3809, 1990a.

Baumjohann, W., Treumann, R. A., and LaBelle, J.: Average Electric Wave Spectra in the Plasma Sheet: Dependence on Ion Density and Ion Beta, J. Geophys. Res., 95, 3811-3817, doi:10.1029/JA095iA04p03811, 1990 b.

Bonnell, J. W., Mozer, F. S., Delory, G. T., Hull, A. J., Ergun, R. E., Cully, C. M., Angelopoulos, V., and Harvey, P. R.: The electric field instrument (EFI) for THEMIS, Space Sci. Rev., 141, 303341, doi:10.1007/s11214-008-9469-2, 2008.

Cattell, C. A. and Mozer, F. S.: Experimental determination of the dominant wave mode in the active near Earth magnetotail, Geo- 
phys. Res. Lett., 13, 221-224, 1986.

Cattell, C. A., Mozer, F. S., Anderson, R. R., Hones Jr., E. W., and Sharp, R. D.: ISEE Observations of the Plasma Sheet Boundary, Plasma Sheet, and Neutral Sheet, 2. Waves, J. Geophys. Res., 91, 5681-5688, 1986.

Cully, C. M., Ergun, R. E., Stevens, K., Nammari, A., and Westfall, J.: The THEMIS digital fields board, Space Sci. Rev., 141, 343355, doi:10.1007/s11214-008-9417-1, 2008.

Daughton, W.: Electromagnteic propoerties of the lower-hybrid drift instability in a thin current sheet, Phys. Plasmas, 10, 31033119, 2003.

Daughton, W., Lapenta, G., Ricci, P.: Nonlinear evolution of the lower-hybrid drift instability in a current sheet, Phys. Rev. Lett., 93, 105004, doi:10.1103/PhysRevLett.93.105004, 2004.

Davidson, R. C., Gladd, N. T., Wu, C. S., and Huba, J. D.: Effects of finite plasma beta on the lower-hybrid-drift instability, Phys. Fluids, 20, 301-310, 1977.

Deng, X., Ashour-Abdalla, M., Zhou, M., Walker, R., El-Alaoui, M., Angelopoulos, V., Ergun, R. E., and Schriver, D.: Wave and particle characteristics of earthward electron injections associated with dipolarization fronts, J. Geophys. Res., 115, A09225, doi:10.1029/2009JA015107, 2010.

Gary, S. P. and Wang, J.: Whistler instability: Electron anisotropy upper bound, J. Geophys. Res., 101, 10749-10754, doi:10.1029/96JA00323, 1996.

Gurnett, D. A., Frank, L. A., and Lepping, R. P.: Plasma Waves in the Distant Magnetotail, J. Geophys. Res., 81, 6059-6071, 1976.

Howes, G. G., Dorland, W., Cowley, S. C., Hammett, G. W., Quataert, E., Schekochihin, A. A., and Tatsuno, T.: Kinetic simulations of magnetized turbulence in astrophysical plasmas, Phys. Rev. Lett., 100, 065004, doi:10.1103/PhysRevLett.100.065004, 2008.

Ji, H., Kulsrud, R., Fox, W., and Yamada, M.: An obliquely propagating electromagnetic drift instability in the lower hybrid frequency range, J. Geophys. Res., 110, A08212, doi:10.1029/2005JA011188, 2005.

Karimabadi, H., Daughton, W., and Quest, K. B.: Role of electron temperature anisotropy in the onset of magnetic reconnection, Geophys. Res. Lett., 31, L18801, doi:10.1029/2004GL020791, 2004.

Kennel, C. F. and Petschek, H. E.: Limit on stably trapped particle fluxes, J. Geophys. Res., 71, 1-28, 1966.

Kepko, L., Spanswick, E., Angelopoulos, V., Donovan, E., McFadden, J., Glassmeier, K.-H., Raeder, J., and Singer, H. J.: Equatorward moving auroral signatures of a flow burst observed prior to auroral onset, Geophys. Res. Lett., 36, L24104, doi:10.1029/2009GL041476, 2009.

Le Contel, O., Pellat, R., and Roux, A.: Self-consistent quasi-static radial transport during the substorm growth phase, J. Geophys. Res., 105, 12929-12944, doi:10.1029/1999JA900498, 2000.

Le Contel, O., Roux, A., Perraut, S., Pellat, R., Holter, Ø., Pedersen, A., and Korth, A.: Possible control of the plasma transport in the near-Earth plasma sheet via current driven Alfvén waves $(f \sim$ $f \mathrm{H}+$ ), J. Geophys. Res., 106, 10817-10827, 2001.

Le Contel, O., Roux, A., Perraut, S., Pellat, R., Robert, P., Chanteur, G., Fontaine, D., Cornilleau-Wehrlin, N., Sauvaud, J.-A., Cully, C., Parks, G., Chua, D., Andr, M., Balogh, A., Fazakerley, A., Rème, H., Nagai, T., Mukai, T., Hayakawa, H., Matsuoka, A., Anderson, R. R., and Matsumoto, H.: Role of the Parallel Cur- rent Instability during Substorms: Theory and Observations, in: Proceeding 6th International Conference on Substorm (ICS-6), edited by: Winglee, R. M., pp. 326-333, 2002.

Le Contel, O., Roux, A., Robert, P., Coillot, C., Bouabdellah, A., de La Porte, B., Alison, D., Ruocco, S., Angelopoulos, V., Bromund, K., Chaston, C. C., Cully, C., Auster, H. U., Glassmeier, K. H., Baumjohann, W., Carlson, C. W., McFadden, J. P., and Larson, D.: First Results of the THEMIS Search Coil Magnetometers, Space Sci. Rev., 141, 509-534, 2008.

Le Contel, O., Roux, A., Jacquey, C., Robert, P., Berthomier, M., Chust, T., Grison, B., Angelopoulos, V., Sibeck, D., Chaston, C. C., Cully, C. M., Ergun, B., Glassmeier, K.-H., Auster, U., McFadden, J., Carlson, C., Larson, D., Bonnell, J. W., Mende, S., Russell, C. T., Donovan, E., Mann, I., and Singer, H.: Quasi-parallel whistler mode waves observed by THEMIS during near-earth dipolarizations, Ann. Geophys., 27, 2259-2275, doi:10.5194/angeo-27-2259-2009, 2009.

Li, W., Thorne, R. M., Angelopoulos, V., Bortnik, J., Cully, C. M., Ni, B., LeContel, O., Roux, A., Auster, U., and Magnes, W.: Global distribution of whistler-mode chorus waves observed on the THEMIS spacecraft, Geophys. Res. Lett., 36, L09104, doi:10.1029/2009GL037595, 2009.

Li, W., Thorne, R. M., Nishimura, Y., Bortnik, J., Angelopoulos, V., McFadden, J. P., Larson, D. E., Bonnell, J. W., Lecontel, O., Roux, A., and Auster, U.: THEMIS analysis of observed equatorial electron distributions responsible for the chorus excitation, $\mathrm{J}$. Geophys. Res., 115, A00F11, doi:10.1029/2009JA014845, 2010.

Liang, J., Spanswick, E., Nicolls, M. J., Donovan, E. F., Lummerzheim, D., and Liu, W.: Multi-instrument observations of soft-electron precipitation and its association with magnetospheric flows, J. Geophys. Res., A06201, doi:10.1029/2010JA015867, 2011.

Liu, W. W. and Liang, J.: Disruption of magnetospheric current sheet by quasi-electrostatic field, Ann. Geophys., 27, 1941-1950, doi:10.5194/angeo-27-1941-2009, 2009.

Ludlam, M., Angelopoulos, V., Taylor, E., Snare, R. C., Means, J. D., Ge, Y. S., Narvaez, P., Auster, H. U., Le Contel, O., Larson, D., and Moreau, T.: The THEMIS Magnetic Cleanliness Program, Space Sci. Rev., 141, 171-184, 2008.

Matsumoto, H., Kojima, H., Miyatake, T., Omura, Y., Okada, M., Nagano, I., and Tsutsui, M.: Electrostatic solitary waves (ESW) in the magnetotail: BEN wave forms observed by GEOTAIL, Geophys. Res. Lett., 21, 2915-2918, 1994.

McFadden, J. P., Carlson, C. W., Larson, D., Ludlam, M., Abiad, R., Elliott, B., Turin, P., Marckwordt, M., and Angelopoulos, V.: The THEMIS ESA plasma instrument and in-flight calibration, Space Sci. Rev., 141, 277-302, doi:10.1007/s11214-008-94402, 2008.

Meredith, N. P., Horne, R. B., Thorne, R. M., and Anderson, R. R.: Survey of upper band chorus and ECH waves: Implications for the diffuse aurora, J. Geophys. Res., 114, A07218, doi:10.1029/2009JA014230, 2009.

Nagai, T., Fujimoto, M., Saito, Y., Machida, S., Terasawa, T., Nakamura, R., Yamamoto, T., Mukai, T., Nishida, A., and Kokubun, S.: Structure and dynamics of magnetic reconnection for substorm onsets with GEOTAIL observations, J. Geophys. Res, 103, 4419-4440, 1998.

Ni, B., Thorne, R. M., Shprits, Y. Y., Orlova, K., and Meredith, N. P.: Chorus-driven resonant scattering of diffuse auroral electrons 
in non-dipolar magnetic fields, J. Geophys. Res., 116, A06225, doi:10.1029/2011JA016453, 2011.

Omura, Y. and Matsumoto, H.: Competing processes of whistler and electrostatic instabilities in the magnetosphere, J. Geophys. Res., 92, 8649-8659, 1987.

Perraut, S., Le Contel, O., Roux, A., Korth, A. Ø., Holter, O., and Pedersen, A.: Disruption of parallel current at substorm breakup, Geophys. Res. Lett., 27, 4041-4044, 2000.

Perraut, S., Le Contel, O., Roux, A., Parks, G., Chua, D., Hoshino, M., Mukai, T., and Nagai, T.: Substorm expansion phase: Observations from Geotail, Polar and IMAGE network, J. Geophys. Res., 108, 1159, doi:10.1029/2002JA009376, 2003.

Pritchett, P. L., and Coroniti, F. V.: Kinetic simulations of 3-D reconnection and magnetotail disruptions, Earth Planets Space, 53, 635-643, 2001.

Roux, A., Le Contel, O., Coillot, C., Bouabdellah, A., de La Porte, B., Alison, D., Ruocco, S., and Vassal, M. C: The search coil magnetometer for THEMIS, Space Sci. Rev., 141, 265-275, doi:10.1007/s11214-008-9455-8, 2008.

Roux, A., Robert, P., Le Contel, O., Angelopoulos, V., Auster, U., Bonnell, J., Cully, C. M., Ergun, R. E., and McFadden, J. P.: A mechanism for heating electrons in the magnetopause current layer and adjacent regions, Ann. Geophys., 29, 2305-2316, doi:10.5194/angeo-29-2305-2011, 2011.

Runov, A., Angelopoulos, V., Sitnov, M. I., Sergeev, V. A., Bonnell, J., McFadden, J. P., Larson, D., Glassmeier, K.H., and Auster, U.: THEMIS observations of an earthwardpropagating dipolarization front, Geophys. Res. Lett., 36, L14106, doi:10.1029/2009GL038980, 2009.

Runov, A., Angelopoulos, V., Sitnov, M., Sergeev, V. A., Nakamura, R., Nishimura, Y., Frey, H. U., McFadden, J. P., Larson, D., Bonnell, J., Glassmeier, K.-H., Auster, U., Connors, M., Russell, C. T., and Singer, H. J.: Dipolarization fronts in the magnetotail plasma sheets, Planet. Space Sci., 59, 517-525, doi:10.1016/j.pss.2010.06.006, 2010.

Runov, A., Angelopoulos, V., Zhou, X.-Z., Zhang, X.-J., Li, S., Plaschke, F., and Bonnell, J.: A THEMIS multi-case study of dipolarization fronts in the magnetotail plasma sheet, J. Geophys. Res., 116, A05216, doi:10.1029/2010JA016316, 2011.

Sagdeev, R. Z., Sotnikov, V. I., Shapiro, V. D., and Shevchenko, V. I.: Contribution to the theory of magnetosonic turbulence, Letters Sov. Phys. JETP, 26, 582-587, 1977.

Sahraoui, F., Goldstein, M. L., Robert, P., and Khotyaintsev, Y. V.: Evidence of a cascade and dissipation of solar-wind turbulence at the electron gyroscale, Phys. Rev. Lett., 102, 231102, doi:10.1103/PhysRevLett.102.231102, 2009.
Schodel, R., Baumjohann, W., Nakamura, R., Sergeev, V. A., and Mukai, T.: Rapid flux transport in the central plasma sheet, J. Geophys. Res., 106, 301-313, doi:10.1029/2000JA900139, 2001, 2001.

Shinohara, I., Nagai, T., Fujimoto, M., Terasawa, T., Mukai, T., Tsuruda, K., and Yamamoto, T.: Low-frequency electromagnetic turbulence observed near the substorm onset site, J. Geophys. Res., 103, 20365, doi:10.1029/98JA01104, 1998.

Sigsbee, K., Cattell, C. A., Fairfield, D., Tsuruda, K., and Kokubun, S.: Geotail observations of low-frequency waves and high-speed earthward flows during substorm onsets in the near magnetotail from 10 to $13 R_{\mathrm{E}}$, J. Geophys. Res., 107, 1141, doi:10.1029/2001JA000166, 2002.

Sitnov, M. I., Lui, A. T. Y., Guzdar, P. N., and Yoon, P. H.: Currentdriven instabilities in forced current sheets, J. Geophys. Res., 109, A03205, doi:10.1029/2003JA010123, 2004.

Volwerk, M., Nakamura, R., Baumjohann, W., Treumann, R. A., Runov, A., Vörös, Z., Zhang, T. L., Asano, Y., Klecker, B., Richter, I., Balogh, A., and Rème, H.: A statistical study of compressional waves in the tail current sheet, J. Geophys. Res., 108, 1429, doi:10.1029/2003JA010155, 2003.

Wilber, M., Lee, E., Parks, G. K., Meziane, K., Carlson, C. W., McFadden, J. P., Rème, H., Dandouras, I., Sauvaud, J.-A., Bosqued, J.-M., Kistler, L., Möbius, E., McCarthy, M., Korth, A., Klecker, B., Bavassano-Cattaneo, M.-B., Lundin, R., and Lucek, E.: Cluster observations of velocity space-restricted ion distributions near the plasma sheet, Geophys. Res. Lett., 31, L24802, doi:10.1029/2004GL020265, 2004.

Zhang, Y., Matsumoto, M., and Omura, Y.: Linear and Nonlinear Interactions of an Electron Beam with Oblique Whistler and Electrostatic Waves in the Magnetosphere, J. Geophys. Res., 98, 21353-21363, 1993.

Zhang, Y., Matsumoto, H., and Kojima, H.: Whistler mode waves in the magnetotail, J. Geophys. Res., 104, 28633-28644, 1999.

Zhou, M., Deng, X. H., Li, S. Y., Pang, Y., Vaivads, A., Rème, H., Lucek., E., Fu, S., Lin, X., Yuan, Z. G., and Wang, J. F.: Observation of waves near lower hybrid frequency in the reconnection region with thin current sheet, J. Geophys. Res., 114, A02216, doi:10.1029/2008JA013427, 2009a.

Zhou, M., Ashour-Abdalla, M., Deng, X., Shriver, D., ElAlaoui, M., and Peng, Y.: THEMIS observation of multiple dipolarization fronts and associated wave characteristics in the near-Earth magnetotail, Geophys. Res. Lett., 36, L20107, doi:10.1029/2009GL040663, 2009b. 